\title{
To Select or to Weigh: A Comparative Study of Linear Combination Schemes for Superparent-One-Dependence Estimators
}

\author{
Ying Yang, Geoffrey I. Webb, Senior Member, IEEE, Jesús Cerquides, Kevin B. Korb, \\ Janice Boughton, and Kai Ming Ting
}

\begin{abstract}
We conduct a large-scale comparative study on linearly combining superparent-one-dependence estimators (SPODEs), a popular family of seminaive Bayesian classifiers. Altogether, 16 model selection and weighing schemes, 58 benchmark data sets, and various statistical tests are employed. This paper's main contributions are threefold. First, it formally presents each scheme's definition, rationale, and time complexity and hence can serve as a comprehensive reference for researchers interested in ensemble learning. Second, it offers bias-variance analysis for each scheme's classification error performance. Third, it identifies effective schemes that meet various needs in practice. This leads to accurate and fast classification algorithms which have an immediate and significant impact on real-world applications. Another important feature of our study is using a variety of statistical tests to evaluate multiple learning methods across multiple data sets.
\end{abstract}

Index Terms-Classification learning, Bayesian probabilistic learning, ensemble learning, model selection, model weighing, superparent-one-dependence estimator (SPODE).

\section{INTRODUCTION}

$\mathrm{E}$ NSEMBLE learning is a popular method in classification learning. It combines multiple learning models' decisions to produce more accurate results than single models [1], [2], [3], [4], [5]. This paper focuses on two particular aspects of ensemble learning, selection, and weighing of models for linear model combination. The goal is to study formally alternative selection or linear weighing schemes in theory and to identify effective and efficient ones for practical use.

The general problem for model selection is, given some sample data, how to decide which are the most effective models within some model space. The general problem of linear model weighing focuses on calculating the weight associated with each model within some model space and accordingly weighing their decisions when ensembling.

This paper looks at the model space of Bayesian network classifiers. In particular, superparent-one-dependence estimators (SPODEs) [6], [7], a popular family of seminaive Bayesian classifiers, are taken as a vehicle of illustration throughout the research.

- Y. Yang, G.I. Webb, K.B. Korb, and J. Boughton are with the Clayton School of Information Technology, Clayton Campus, Monash University, Clayton, VIC 3800, Australia. E-mail: \{ying.yang, geoff.webb, kevin.korb, janice.boughton\}@infotech.monash.edu.au.

- J. Cerquides is with the Departament de Matemàtica Aplicada y Anàisi, Universitat de Barcelona, C/Gran Via, 585, Barcelona, 08007 Spain. E-mail: cerquide@maia.ub.es.

- K.M. Ting is with the Gippsland School of Information Technology, Gippsland Campus, Monash University, Churchill, VIC 3842, Australia. E-mail: kaiming.ting@infotech.monash.edu.au.

Manuscript received 12 Oct. 2006; revised 3 Mar. 2007; accepted 8 May 2007; published online 9 Aug. 2007.

For information on obtaining reprints of this article, please send e-mail to: tkde@computer.org, and reference IEEECS Log Number TKDE-0473-1006.

Digital Object Identifier no. 10.1109/TKDE.2007.190650.
This paper presents 16 alternative model selection or weighing schemes. Selection schemes include Akaike's information criterion (AIC), Bayesian information criterion (BIC), minimum description length (MDL), minimum message length (MML), random selection (RAN), cross validation $(\mathrm{CV})$, forward sequential addition (FSA), backward sequential elimination (BSE), lazy elimination (LE). Weighing schemes include AIC, BIC, MDL, MML, Bayesian model averaging (BMA), maximum a posteriori linear mixture of discriminative distributions (MAPLMD), and maximum a posteriori linear mixture of generative distributions (MAPLMG). A large-scale empirical comparison using 58 benchmark data sets is conducted to test the classification accuracy and efficiency of ensembles that result from using alternative schemes. A variety of statistics are employed to thoroughly evaluate and rank their performances.

By doing this research, we seek answers to the following questions:

1. What are every scheme's strength and weakness for ensemble learning?

2. Which scheme is consistently among the best algorithms for our large suite of data sets?

3. In general, which is more effective and/or more efficient, model selection or model weighing?

4. How to choose which scheme to use in practice?

\section{BACKGROUND}

This section defines the terminology and notation that will be used throughout this paper. It also explains how a SPODE and an ensemble of SPODEs carry out classification. 


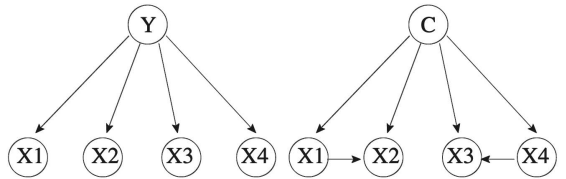

(a)

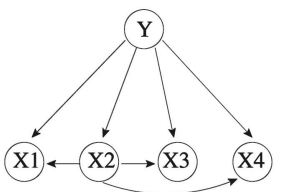

(c)
Fig. 1. Illustration of (a) NB, (b) ODE, and (c) SPODE. An arc points from a parent to a child. A child only depends on its parents. NB assumes that each attribute only depends on the class $Y$ and is independent of other attributes given the class. ODE allows each attribute to depend on at most one other attribute in addition to the class. SPODE assumes that each attribute can depend on a common attribute (the superparent $X_{2}$ ) in addition to the class.

\subsection{Terminology and Notation}

This paper addresses the problem of classification learning using Bayesian network classifiers. The following terminology and notation will be used.

An instance $\mathbf{x}\left\langle x_{1}, x_{2}, \cdots, x_{m}\right\rangle$ is a vector of $m$ attribute values $x_{i}$, each observed for an attribute variable $X_{i}(i \in[1, m])$. As SPODEs currently require discrete-valued data, numeric attributes are discretized. An instance can also have a class label $y$ corresponding to the class variable $Y$. If its class label is known, an instance is labeled. Otherwise, it is unlabeled. Whenever applicable, for the purpose of uniformity in formulas, $X_{i}$ represents the class variable when $i=m+1$. Training data $D$ is a set of labeled instances from which a classifier is learned to predict the class labels of unlabeled instances. The number of training instances is $n$. The number of values for $X_{i}$ is $v_{i}$. $X_{i}$ 's parent variables are $\Phi(i)$. The number of joint states (joint instantiated values) of parents of $X_{i}$ is $|\phi(i)|$. The $r$ th joint state of the parents is $\phi_{i r}$. When applicable, $h$ indicates a SPODE in general, and $h_{i}$ indicates a particular SPODE whose superparent is $X_{i}$. Generally, the $\log$ base in information metrics does not matter. A common practice is to use $e$ or 2 .

\subsection{SPODE}

Bayesian network classifiers have long been a core technique in predictive learning. The naive Bayesian (NB) classifier is among the first Bayesian networks introduced into machine learning. NB assumes attributes conditionally independent of each other given the class. It is very efficient with reasonable prediction accuracy [8], [9], [10], [11], [12], [13], [14], [15]. In recent years, there has also been considerable interest in developing variants of NB that weaken the attribute independence assumption in order to further improve the prediction accuracy [6], [7], [16], [17], [18], [19], [20], [21], [22], [23], [24], [25], [26], [27], [28], [29], [30]. For instance, one-dependence estimators (ODEs) [23] such as the tree-augmented naive Bayes (TAN) [16] provide a powerful alternative to NB. As depicted in Fig. 1, an ODE is similar to an NB except that each attribute is allowed to depend on at most one other attribute in addition to the class. Among ODEs, SPODEs [6], [7] have received a lot of attention because they offer a combination of high training efficiency, high classification efficiency, and high classification accuracy [30], [31], [32], [33], [34], [35], [36], [37], [38], [39], [40], [41], [42], [43], [44], [45], [46], [47]. Those merits give SPODEs a great potential to substitute for naive Bayes classifiers in numerous real-world classification systems, including medical diagnosis, fraud detection, e-mail filtering, document classification, and Web page prefetching. As illustrated in Fig. 1, a SPODE relaxes NB's attribute independence assumption by allowing all attributes to depend on a common attribute, the superparent, in addition to the class.

To classify an instance $\mathbf{x}$, a Bayesian network classifier calculates $\hat{P}(y \mid \mathbf{x})$ for each $y \in Y$, an estimate of the probability of the class label given this instance $P(y \mid \mathbf{x})$. The label attaining the highest probability will be assigned to $\mathbf{x}$. Since $P(y \mid \mathbf{x})=\frac{P(y, \mathbf{x})}{P(\mathbf{x})}$ and $P(\mathbf{x})$ is invariant across different class labels, one only needs to estimate $P(y, \mathbf{x})$ as

$$
\operatorname{argmax}_{y} P(y \mid \mathbf{x})=\operatorname{argmax}_{y} P(y, \mathbf{x}) .
$$

A SPODE with superparent $X_{p}$ uses (2) to calculate $\hat{P}(y, \mathbf{x})$. The second equation results from SPODEs' assumption that all attributes are independent of each other given the class $Y$ and the superparent $X_{p}$ :

$$
\begin{aligned}
\hat{P}(y, \mathbf{x}) & =\hat{P}\left(y, x_{p}\right) \hat{P}\left(\mathbf{x} \mid y, x_{p}\right) \\
& =\hat{P}\left(y, x_{p}\right) \prod_{i=1}^{m} \hat{P}\left(x_{i} \mid y, x_{p}\right) .
\end{aligned}
$$

\subsection{SPODE Ensemble}

There has been a strong interest in ensembling SPODEs because it can decrease a single SPODE's classification variance and attain high classification accuracy with moderate time requirement [30], [31], [32], [33], [34], [35], [36], [37], [38], [39], [40], [41], [42], [43], [44], [45], [46], [47].

For a training data set with $m$ attributes, there can be $m$ candidate SPODEs, each taking a different attribute as its superparent. A SPODE ensemble is a linear combination of multiple SPODEs' probability estimates. It classifies $\mathrm{x}$ using the following equation, where each $\hat{P}_{j}(y, \mathbf{x})$ is calculated by a SPODE using (2) with $p=j$ :

$$
\hat{P}(y, \mathbf{x})=\sum_{j=1}^{m} w_{j} \hat{P}_{j}(y, \mathbf{x}) .
$$

The first approach to ensembling SPODES used equal weight combination of all SPODEs whose parent value occurred above a user-specified minimum frequency in the training data [30]. Subsequent research suggested that frequency is not a useful model selection criterion and that appropriate weighing can substantially improve upon equal weighing, such as in the MAPLMD and MAPLMG weighing schemes [31]. On the other hand, it has also been shown that model selection can be effective when ensembling SPODEs [35], [44]. This paper presents a comprehensive investigation into the relative merits of alternative approaches to weighing and selecting.

\section{Model Selection Schemes}

The general problem for model selection is, given some sample data, how to decide which are the most effective models within some model space. This paper looks at the 
space of SPODE models. Only selected SPODEs will be included in the ensemble. Strictly speaking, model selection is an extreme form of model weighing where the weights are either 1 or 0 . That is,

$$
w_{j}= \begin{cases}1 & \text { if } \mathrm{SPODE}_{j} \text { is selected } \\ 0 & \text { otherwise. }\end{cases}
$$

However, because information-theoretic schemes take different forms when used in model selection versus weighing, this study differentiates selection from weighing.

\subsection{Information-Theoretic Metrics}

Information-theoretic metrics, including AIC, BIC, MDL, and MML [48], [49], [50], [51], provide a combined score, as in (4), for a proposed explanatory model (a SPODE in our context) and for the data given the model. They aim to find a balance between the goodness of fit (minimizing $I(D \mid h)$ ) and model simplicity (minimizing $I(h)$ ) and thereby achieve good modeling performance without overfitting the data. The best score is the smallest. Hence, the lower the score a SPODE gets, the higher its priority to appear in the ensemble:

$$
\text { score }=I(D \mid h)+I(h) .
$$

The term $I(D \mid h)$ is shared by information-theoretic metrics and is

$$
I(D \mid h)=n\left(\sum_{i=1}^{m+1} H\left(X_{i}\right)-\sum_{i=1}^{m+1} H\left(X_{i}, \Phi(i)\right)\right),
$$

where $H\left(X_{i}\right)$ is the entropy of $X_{i}$, and $H\left(X_{i}, \Phi(i)\right)$ is the mutual information between $X_{i}$ and its parents:

$$
\begin{gathered}
H\left(X_{i}\right)=-\sum_{j=1}^{v_{i}}\left(P\left(X_{i}=x_{i j}\right) \log P\left(X_{i}=x_{i j}\right)\right), \\
H\left(X_{i}, \Phi(i)\right)=\sum_{j=1}^{v_{i}} \sum_{r=1}^{\left|\phi_{i}\right|}\left(P\left(x_{i j}, \phi_{i r}\right) \log \frac{P\left(x_{i j}, \phi_{i r}\right)}{P\left(x_{i j}\right) P\left(\phi_{i r}\right)}\right) .
\end{gathered}
$$

How to compute $I(h)$ varies among different schemes and is presented below:

a. Akaike's Information Criterion (AIC). According to Akaike [48],

$$
I_{A I C}(h)=2\left(\sum_{i=1}^{m+1}\left(v_{i}-1\right) \prod_{j \in \Phi(i)} v_{j}\right) .
$$

For any root node $X_{i}$ (where $\Phi(i)=\emptyset$ ), the product term on the right should be replaced by 1 . The same principle also applies to BIC and MDL below.

b. Bayesian Information Criterion (BIC). According to Schwarz [49],

$$
I_{B I C}(h)=(\log n)\left(\sum_{i=1}^{m+1}\left(v_{i}-1\right) \prod_{j \in \Phi(i)} v_{j}\right) .
$$

c. Minimum Description Length (MDL). According to Suzuki [50],

$$
I_{M D L}(h)=\left(\frac{1}{2} \log n\right)\left(\sum_{i=1}^{m+1}\left(v_{i}-1\right) \prod_{j \in \Phi(i)} v_{j}\right) .
$$

d. Minimum Message Lenth (MML). According to Korb and Nicholson [51],

$$
\begin{aligned}
I_{M M L}(h)= & \log (m+1) !+C_{2}^{m+1}-\log (m-1) ! \\
& +\sum_{i=1}^{m+1} \frac{v_{i}-1}{2}\left(\log \frac{\pi}{6}+1\right) \\
& -\log \prod_{i=1}^{m+1} \prod_{j=1}^{\left|\phi_{i}\right|}\left(\frac{\left(v_{i}-1\right) !}{\left(S_{i j}+v_{i}-1\right) !} \prod_{l=1}^{v_{i}} \alpha_{i j l} !\right),
\end{aligned}
$$

where $S_{i j}$ is the number of training instances where the parents $\Phi(i)$ take their joint $j$ th value, and $\alpha_{i j l}$ is the number of training instances where $X_{i}$ takes its $l$ th value and $\Phi(i)$ take their $j$ th joint value. For any root $X_{i},\left|\phi_{i}\right|$ should be treated as 1 , and every instance should be treated as matching the parents for the purposes of computing $S_{i j}$ and $\alpha_{i j l}$. Equation (10) looks complicated, but it can be computed in polynomial time [52].

Each information-theoretic metric can order a sequence of SPODEs by their supposed merits. One should then expect that excluding poorly predictive SPODEs could improve the classification accuracy. For instance, after it has reached the optimal classification accuracy, an ensemble should not proceed to include additional SPODEs that are counterproductive, even when there are some left. To decide when SPODEs of sufficient merit are no longer to be found for the ensemble given an ordered sequence of $m$ SPODEs, $m$ ensembles are tested. Starting with an empty ensemble, each ensemble in turn includes further one SPODE in the queue. Every ensemble's leave-one-out CV accuracy is calculated. The ensemble with the lowest error is the one to be selected.

\subsection{Random Selection (RAN)}

RAN randomly orders SPODEs. Following the practice with information-theoretic metrics, it then tests $m$ ensembles from size 1 to size $m$, and the one with the lowest leave-oneout CV error is selected. RAN has low computational overhead and offers a useful comparator against which to judge the impact on classification error of other selection schemes.

\subsection{Cross Validation (CV)}

CV [35] scores each individual SPODE by its CV error in the training data. Particularly, in this study, leave-one-out $\mathrm{CV}$ is employed. Given a SPODE, CV loops through the training data $n$ times, each time training the SPODE from $(n-1)$ instances to classify the remaining one instance. The misclassifications are summed and averaged over $n$ iterations. The resulting classification error rate is taken as the metric value of the SPODE. The lower the metric, 
the higher the priority for the SPODE to be used. This process is very efficient as the model need only be updated for each instance that is left out, rather than recalculated from scratch.

Following the practice with information-theoretic metrics, after CV orders SPODEs according to their merits, it tests $m$ ensembles from size 1 to size $m$, and the one with the lowest leave-one-out CV error is selected.

\subsection{Forward Sequential Addition (FSA)}

Inspired by the forward sequential selection strategy for attribute selection in NB [21], FSA [35] begins with an empty ensemble. It then uses hill-climbing search to iteratively add SPODEs most helpful for lowering the ensemble's classification error. In each iteration, suppose the current ensemble is $E_{\text {current }}$ with $k$ SPODEs. FSA in turn adds each candidate SPODE, one that has not been included into $E_{\text {current }}$, and obtains an ensemble $E_{\text {test }}$ of size $(k+1)$. It then calculates the leave-one-out $\mathrm{CV}$ error of $E_{\text {test }}$. The $E_{\text {test }}$ who obtains the lowest error is retained. The corresponding added SPODE is permanently included into the ensemble and deleted from the candidate list. The same process is applied to the new SPODE ensemble of size $(k+1)$ and so on, until every SPODE has been included. The order of addition produces a ranking order for SPODEs. The earlier a SPODE is added, the more merit it possesses and the higher its priority to be used.

The ensemble that achieves the lowest leave-one-out CV error in training during the addition process is selected. If multiple ensembles attain the lowest error, the one that includes the most SPODEs is chosen, as a means to reduce variance caused by model selection [30].

\subsection{Backward Sequential Elimination (BSE)}

Inspired by the BSE strategy for attribute selection in NB [21], BSE [35] starts out with a full ensemble including every SPODE. It then uses hill-climbing search to iteratively eliminate SPODEs whose individual exclusion is most helpful for lowering the classification error. In each iteration, suppose the current ensemble is $E_{\text {current }}$ involving $k$ SPODEs. BSE eliminates each member SPODE in turn from $E_{\text {current }}$ and obtains an ensemble $E_{\text {test }}$ of size $(k-1)$. It then calculates the leave-one-out $\mathrm{CV}$ error of $E_{\text {test }}$. The $E_{\text {test }}$. that yields the lowest error is retained. The corresponding eliminated SPODE is permanently deleted from the ensemble. The same process is applied to the new SPODE ensemble of size $(k-1)$ and so on, until the ensemble is empty. The order of the elimination produces a ranking order for SPODEs. The earlier a SPODE is eliminated, the less merit it possesses and the lower its priority to be used.

The ensemble that achieves the lowest leave-one-out CV error in training during the elimination process is selected. If multiple ensembles attain the lowest error, the one that includes the most SPODEs is chosen, as a means to reduce variance caused by model selection [30].

\subsection{Lazy Elimination (LE)}

The above schemes studied so far select at training time a subset of SPODEs that are used to classify all test instances. An alternative approach delays selection until classification time. LE [44] is based on the observation that $\forall a, b, c: P(a \mid b)=1.0$ entails $P(c \mid a, b)=P(c \mid b)$. Hence, if it can be inferred that one attribute value entails another, assuming conditional independence between the values is likely to be harmful, and the more general value $a$ may safely be deleted. To this end, before a test instance is classified, LE deletes any attribute value $x_{i}$ of the instance that occurs in the training data more than a user-defined minimum number of times (in this research, $30)$ and for which there is another value $x_{j}(j \neq i)$ such that $x_{i}$ is present in every training instance containing $x_{j}$. If $x_{i}$ and $x_{j}$ are identical, only one is deleted. Effectively, LE performs lazy selection by not using SPODEs whose superparents are generalizations of other values of the instance to be classified. Note however that it also deletes children from within SPODEs and hence is not solely a SPODE selection algorithm.

\section{Linear Model Weighing Schemes}

Linear model weighing focuses on calculating the weight associated with each SPODE to linearly combine their probability estimates of $P(y, \mathbf{x})$, as in (3).

\subsection{Information-Theoretic Metrics}

Since the information-theoretic metrics AIC, BIC, MDL, and MML, as defined in Section 3.1, rely upon the Shannon information theory [53] for their motivation and interpretation, it is appropriate to ask what kind of probabilistic weight they imply for the purpose of prediction. In principle, they should support the inversion of Shannon's law to derive the posterior probability of a model given the data for such purposes. Hence, the weight $w$ for a SPODE $h$ is

$$
\begin{aligned}
w & =\hat{P}(h \mid D), \\
& =e^{-I(h \mid D)}, \\
& =e^{-(I(D \mid h)+I(h)-I(D))},
\end{aligned}
$$

where $I(D)=n \sum_{i=1}^{m+1} H\left(X_{i}\right)$ is the entropy of data whose $H\left(X_{i}\right)$ is calculated by (6), $I(D \mid h)$ is calculated by (5), and $I(h)$ is calculated by (7), (8), (9), and (10), respectively, for AIC, BIC, MDL, and MML to be weights.

\subsection{Bayesian Model Averaging (BMA)}

BMA [54], [55] is theoretically the optimal method for combining learned models. It provides a coherent mechanism to ensemble classification models by accounting for single models' uncertainty of generating the data. In the Bayesian view, using a single model to make predictions ignores the uncertainty caused by training data as to which is the correct model; thus, all possible models in the model space under consideration should be used when making predictions, with each model weighted by its probability of being the correct model $P\left(h_{i} \mid D\right)$.

Given an instance $\mathbf{x}$ and a set of classifiers $h_{i}$, BMA estimates the probability of each class label given $\mathrm{x}$ using

$$
\hat{P}(y \mid \mathbf{x})=\sum_{i=1}^{m} \hat{P}\left(y \mid h_{i}\right) \hat{P}\left(h_{i} \mid D\right),
$$


where $\hat{P}\left(y \mid h_{i}\right)$ is the class probability estimated by a SPODE, as in (2). One common approach to estimating the weight was proposed by Cooper and Herskovits [52]:

$$
w_{i}=\hat{P}\left(h_{i} \mid D\right)=\frac{\hat{P}\left(h_{i}, D\right)}{\sum_{i=1}^{m} \hat{P}\left(h_{i}, D\right)},
$$

where

$$
\begin{aligned}
\hat{P}\left(h_{i}, D\right) & =\hat{P}\left(h_{i}\right) \prod_{k=1}^{m+1} \prod_{j=1}^{\left|\phi_{i}\right|}\left(\frac{\left(v_{k}-1\right) !}{\left(S_{k j}+v_{k}-1\right) !} \prod_{l=1}^{v_{k}} \alpha_{k j l} !\right) \\
\hat{P}\left(h_{i}\right) & =\frac{1}{m} \text { if there are } m \text { candidate SPODEs }
\end{aligned}
$$

and $S_{k j}$ and $\alpha_{k j l}$ have the same meanings as in (10).

\subsection{Maximum a Posteriori Linear Mixture of Generative Distributions (MAPLMG)}

The method of maximum a posteriori (MAP, or posterior mode) estimation can be used to obtain a point estimate of an unobserved quantity on the basis of empirical data. It is closely related to Fisher's method of maximum likelihood (ML) but employs an augmented optimization objective that incorporates a prior distribution over the quantity one wants to estimate. MAPLMG and MAPLMD both assume as a prior distribution a Dirichlet over the SPODE ensemble weights. Once this is done, they use MAP estimation to find the most probable set of weights for a SPODE ensemble given a concrete data set. The difference between MAPLMG and MAPLMD is that the former finds the MAP weights for an ensemble of generative probabilistic models, whereas the latter finds the MAP weights for an ensemble of discriminative probabilistic models.

MAPLMG [31] constructs a SPODE ensemble that maximizes the supervised posterior probability of the weights given the training data. It determines the weighing vector $\mathbf{w}\left\langle w_{1}, \ldots, w_{m}\right\rangle$ as

$$
\mathbf{w}=\operatorname{argmax}_{\mathbf{w}} \hat{P}_{L M G}(\mathbf{w} \mid D),
$$

where

$$
\hat{P}_{L M G}(\mathbf{w} \mid D)=\prod_{\langle\mathbf{x}, y\rangle \in D}\left(\frac{\sum_{i=1}^{m} w_{i} \hat{P}_{i}^{L O O}(y, \mathbf{x})}{\sum_{y \in Y} \sum_{i=1}^{m} w_{i} \hat{P}_{i}^{L O O}(y, \mathbf{x})} \prod_{i=1}^{m} w_{i}\right)
$$

and $\hat{P}_{i}^{L O O}(y, \mathbf{x})=\hat{P}\left(x_{i}, y\right) \prod_{j=1}^{m} \hat{P}\left(x_{j} \mid x_{i}, y\right)$, whose right-hand side is estimated from $(D-\{\langle\mathbf{x}, y\rangle\})$ for $h_{i}$. The maximization appearing in (14) is a constrained nonlinear optimization problem that can be solved by means of a sequence of unconstrained maximizations [56], each of them solved by a Newton-like optimization procedure such as the BroydenFletcher-Goldfarb-Shanno (BFGS) method [57].

\subsection{Maximum a Posteriori Linear Mixture of Discriminative Distributions (MAPLMD)}

A scheme closely related to MAPLMG is MAPLMD. It also constructs a SPODE ensemble that maximizes the supervised posterior probability of the weights. It differs from MAPLMG in that the ensemble constructed linearly combines $\hat{P}_{i}(y \mid \mathbf{x})$ instead of $\hat{P}_{i}(y, \mathbf{x})$ in (3):

$$
\hat{P}(y \mid \mathbf{x}) \approx \sum_{i=1}^{m} w_{i} \hat{P}_{i}(y \mid \mathbf{x}) .
$$

It determines weights as

$$
\mathbf{w}=\operatorname{argmax}_{\mathbf{w}} \hat{P}_{L M D}(\mathbf{w} \mid D),
$$

where

$$
\hat{P}_{L M D}(\mathbf{w} \mid D) \propto \prod_{\langle\mathbf{x}, y\rangle \in D}\left(\sum_{i=1}^{m} w_{i} \hat{P}_{i}^{L O O}(y \mid \mathbf{x}) \prod_{i=1}^{m} w_{i}\right)
$$

and $\hat{P}_{i}^{L O O}(y \mid \mathbf{x})$ is $h_{i}$ 's probability estimate for $\mathbf{x}^{\prime}$ s true class given $(D-\{\langle\mathbf{x}, y\rangle\})$. The maximization appearing in (15) can be computed by means of the Expectation-Maximization (EM) algorithm [58].

\section{Time Complexity Analysis}

Assume that the number of training instances and attributes are $n$ and $m$, and the number of classes is $c$. Let the average number of values for an attribute be $v$.

\subsection{Training Overhead}

The time complexity of each scheme to order SPODEs by their merits or to calculate their weights is as follows.

\subsubsection{AIC, BIC, and MDL}

The complexity of calculating $I(D \mid h)$ is $O\left(m v^{2} c\right)$. The dominating part is from $H\left(X_{i}, \Phi(i)\right)$, which iterates through every attribute $(O(m))$, then every value $(O(v))$, and then every joint value of the superparent and the class $(O(v c))$. The complexity of calculating $I(h)$ is $O(m) .{ }^{1}$ Since the selection repeats for each attribute $(O(m))$, the overall complexity is $O\left(m \times\left(m v^{2} c+m\right)\right)=O\left(m^{2} v^{2} c\right)$.

\subsubsection{MML and BMA}

The dominating complexity of MML, as well as BMA, for SPODEs is from

$$
\prod_{i=1}^{m+1} \prod_{j=1}^{\left|\phi_{i}\right|} \frac{\left(v_{i}-1\right) !}{\left(S_{i j}+v_{i}-1\right) !} \prod_{l=1}^{v_{i}} \alpha_{i j l} !
$$

MML iterates through each attribute $(O(m))$ and then each joint value of the superparent and the class $(O(v c))$ for which two factorials are calculated $\left(O(v)+O\left(\frac{n}{v c}\right)\right)$. On top of that, it loops through each attribute value $(O(v))$ for which a third factorial is calculated $\left(O\left(\frac{n}{v^{2} c}\right)\right)$. Hence, the complexity is $O\left(m * v c *\left(v+\frac{n}{v c}\right) * v * \frac{n}{v^{2} c}\right)=O\left(m n\left(v+\frac{n}{v c}\right)\right)$. This repeats for each attribute $(O(m))$, and the overall complexity is hence $O\left(m^{2} n\left(v+\frac{n}{v c}\right)\right)$.

\subsubsection{CV}

To classify an instance, a SPODE will multiply the conditional probability of each attribute value given each class label and one (constant) superparent value. This results in $O(m c)$. To do leave-one-out $\mathrm{CV}$, the classification will repeat $n$ times. Hence, the complexity is $O(m c n)$. This

1. Although MDL has an extra loop $\prod_{j \in \Phi(i)} v_{j}$, in case of a SPODE, $|\Phi(i)|$ is of maximum value 2 (the superparent and the class). Hence, it can be treated as a constant and does not increase the order of the complexity. 
repeats for each attribute $(O(m))$, and the overall complexity is hence $O\left(m^{2} c n\right)$.

\subsubsection{FSA}

The hill-climbing procedure of increasing a SPODE ensemble from being empty to size $m$ will render a complexity of $O\left(\mathrm{~m}^{2}\right)$. In the first round, it alternatively adds each of $m$ SPODEs. In the second round, it alternatively adds each of $(m-1)$ SPODEs. Following this line of reasoning, the total number of probing a SPODE is $m+(m-1)+\cdots+2+1=O\left(m^{2}\right)$. As explained for $\mathrm{CV}$, testing each SPODE by leave-one-out CV will incur a complexity of $O(m c n)$. As a result, the overall complexity is $O\left(m^{3} \mathrm{cn}\right)$.

\subsubsection{BSE}

The hill-climbing procedure of reducing a SPODE ensemble of size $m$ to 0 will render a complexity of $O\left(m^{2}\right)$. In the first round, it alternatively eliminates each of $m$ SPODEs. In the second round, it alternatively eliminates each of $(m-1)$ SPODEs. Following this line of reasoning, the total number of probing a SPODE is $m+(m-1)+\cdots+2+1=O\left(m^{2}\right)$. As explained for $\mathrm{CV}$, testing each SPODE by leave-one-out $\mathrm{CV}$ will incur a complexity of $O(m c n)$. As a result, the overall complexity is $O\left(m^{3} \mathrm{cn}\right)$.

\subsubsection{LE}

LE does not require any additional information to be gathered at training time and hence has no impact on training time.

\subsubsection{MAPLMD}

The computation of the optimal weights can be implemented in two steps. In the first step, $P_{i}^{L O O}(y \mid \mathbf{x})$ of each $h_{i}$ is computed for each training instance. This takes $O\left(\mathrm{~m}^{2} \mathrm{cn}\right)$, as reasoned in Section 5.1.3. After that, the EM algorithm iterates until convergence or until the maximum number of 10,000 iterations is reached. Each EM iteration takes $O(n m c)$. The complete computational complexity is therefore $O\left(m^{2} c n+K m n c\right)$, where $\mathrm{K}$ is the bound of the number of iterations in the maximization algorithm. Since $\mathrm{K}$ is fixed, it does not affect the theoretical computational complexity but influences the computing time when $m, n$ and $c$ are not relatively large enough. Hence, we keep the large constant $K$ in the complexity expression.

\subsubsection{MAPLMG}

The computation of the optimal weights can be implemented in two steps. In the first step, $P_{i}^{L O O}(y, \mathbf{x})$ of each $h_{i}$ is computed for each training instance. This takes $O\left(\mathrm{~m}^{2} \mathrm{cn}\right)$, as reasoned in Section 5.1.3. After that, the maximum is found by a sequence of applications of the BFGS minimization algorithm until convergence or the maximum number of 1,000 iterations is reached. Each BFGS iteration computes both the value of the function it tries to maximize and the value of its derivative. In this case, this can be done in $O(n m c)$. Following the same reasoning as for the above MAPLMD, the complete computational complexity is therefore $O\left(m^{2} c n+K m n c\right)$.

\subsection{Classification Overhead}

For selection schemes, the result is a linear combination of SPODEs. Hence, each scheme's complexity is of the same order $O\left(m^{2} c\right)$, resulting from the $O(m c)$ SPODE algorithm applied over an $O(m)$ sized ensemble. Please note that LE requires a test each time a pair of attribute values is considered to determine whether one is a generalization of the other, incurring an additional complexity $O\left(\mathrm{~m}^{2}\right)$.

For weighing schemes, following the above lines of reasoning, its classification complexity is $O\left(\mathrm{~m}^{2} \mathrm{c}\right)$. More precisely, the weighing's complexity is higher than that of selection's by $\mathrm{O}(1)$, resulting from multiplying each SPODE's probability estimate by its weight.

\section{EXPERIMENTS}

Empirical tests, observations, analyses, and evaluations are presented here for each selection or weighing scheme. The objective function is to maximize the learning accuracy and efficiency of the resulting ensemble classifiers. The Averaged One-Dependence Estimator (AODE) [30], a complete SPODE ensemble without any selection or weighing applied, is also included to offer a baseline in comparing alternative schemes.

\subsection{Data}

Rival schemes are implemented in the Waikato Environment for Knowledge Analysis (WEKA) machine learning environment [59] and are validated using a large suite of 58 benchmark data sets from the UCI machine learning repository and KDD archive [60], as described in Table 1. Because SPODEs currently require discrete-valued data, numeric attributes are discretized using the WEKA MDL discretizer [59]. Since part of the software (information metrics) does not handle missing values, following WEKA's practice, missing values for nominal and numeric attributes in a data set are replaced with the modes and means, respectively.

\subsection{Design}

Each scheme is tested on each data set using a 30-trial twofold CV. An $s$-fold CV divides a data set into $s$ equalsized subsets. Each subset is used in turn as a test set with the remaining $(s-1)$ data sets used for training. One may conduct $s$-fold $\mathrm{CV}$ for $t$ trials, each trial shuffling the instances and forming $s$ different subsets. The reason that we use a substantial number (30) of trials is because we perform bias-variance decomposition analysis, which is more accurate when sufficient trials are conducted [61]. The reason that we use a twofold $\mathrm{CV}$ is to maximize the variation in the training data from trial to trial.

Five performance measures are recorded on each data set: training time, classification time, and classification error, which can be decomposed into a bias term and a variance term [61], [62], [63], [64], [65]. A third irreducible term is the error of an optimal algorithm (the level of noise in the data). In our study, following Kohavi and Wolpert's method, it is merged into bias [64].

Please note that varying from our previous research, we no longer impose a frequency threshold on SPODEs. Previously, as a means to reduce classification variance, a SPODE was considered a candidate for ensembling only if the parent value's frequency was above 30 [35]. However, 
TABLE 1

Statistics of Experimental Data Sets

\begin{tabular}{|c|c|c|c|c|c|c|c|c|}
\hline Data set & Ins. & Att. & Data & Ins. & Att. & Data & Ins. & Att. \\
\hline Abalone & 4177 & 8 & Ionosphere & 351 & 34 & PrimaryTumor & 339 & 17 \\
\hline JapaneseVowels & 9961 & 12 & IrisPlant & 150 & 4 & Promoter & 106 & 57 \\
\hline Annealing & 898 & 38 & KRvsKP & 3196 & 36 & Satellite & 6435 & 36 \\
\hline Audiology & 226 & 69 & LaborRelations & 57 & 16 & ImageSegmentation & 2310 & 19 \\
\hline Automobile & 205 & 25 & LEDDisplay & 1000 & 7 & SickEuthyroid & 3772 & 29 \\
\hline BalanceScale & 625 & 4 & LetterRecognition & 20000 & 16 & AustralianSignLanguage & 12546 & 8 \\
\hline Bands & 539 & 36 & LiverDisorders & 345 & 6 & Sonar & 208 & 60 \\
\hline BreastCancer & 699 & 9 & LungCancer & 32 & 56 & Soybean & 683 & 35 \\
\hline Chess & 551 & 39 & Lymphography & 148 & 18 & Spambase & 4601 & 57 \\
\hline ContraceptiveMethodChoice & 1473 & 9 & MultipleFeaturesMorphological & 2000 & 6 & SpliceJunction & 3177 & 60 \\
\hline CreditScreening & 690 & 15 & Mushrooms & 8124 & 22 & SyntheticControl & 600 & 60 \\
\hline Echocardiogram & 131 & 6 & Musk & 476 & 166 & Thyroid & 9169 & 29 \\
\hline German & 1000 & 20 & NettalkPhoneme & 5438 & 7 & TicTacToe & 958 & 9 \\
\hline GlassIdentification & 214 & 9 & NewThyroid & 215 & 5 & Vehicle & 846 & 18 \\
\hline HeartDiseaseCleveland & 303 & 13 & OpticalDigits & 5620 & 48 & Vowel & 990 & 11 \\
\hline Hepatitis & 155 & 19 & PageBlocks & 5473 & 10 & Waveform & 5000 & 40 \\
\hline HorseColic & 368 & 21 & PenBasedRecognition & 10992 & 16 & Wine & 178 & 13 \\
\hline CongressionalVoting & 435 & 16 & PimaIndiansDiabetes & 768 & 8 & Yeast & 1484 & 8 \\
\hline HeartDiseaseHungarian & 294 & 13 & Postoperative & 90 & 8 & Zoo & 101 & 16 \\
\hline Hypothyroid & 3772 & 29 & & & & & & \\
\hline
\end{tabular}

"Ins." and "Att." are the number of instances and attributes, respectively.

subsequent research demonstrated better results when the minimum frequency was reduced to 1 [31]. Accordingly, some experimental results differ from those obtained in previous otherwise equivalent experiments [35].

\subsection{Bias-Variance Decomposition of Error}

It is useful to look into the bias and variance of a classifier because they each offer a different perspective on classification error. Bias describes the component of error that results from the systematic error of the learning algorithm. Variance describes the component of error that results from random variation in the training data and from random behavior in the learning algorithm and thus measures how sensitive an algorithm is to changes in the training data. As the algorithm becomes more sensitive, the variance increases.

Moore and McCabe [66] illustrated bias and variance through shooting arrows at a target, as reproduced in Fig. 3. We can think of the perfect model as the bull's-eye on a target, and the learned classifier as an arrow fired at the bull's-eye. Bias and variance describe what happens when an archer fires many arrows at the target. Bias means that the aim is off, and the arrows land consistently off the bull's-eye in the same direction. A large variance means that repeated shots are widely scattered on the target. They do not give similar results but differ widely among themselves. A good learning scheme, like a good archer, should have both a low bias and a low variance. We use Kohavi and Wolpert's definitions of bias and variance [64]. Each instance is classified once in each trial and, hence, 30 times in all.

\subsection{Statistics}

A variety of statistics are employed to evaluate the measured performance of each competing scheme.

- Mean of ranks. Following the practice of Friedman [67], [68], for each data set, we rank competing algorithms. The one that attains the best performance is ranked 1 , the second best is ranked 2, and so forth. A method's mean rank is obtained by averaging its ranks across all data sets. Compared with mean value (the arithmetic mean of measured performance, such as error, across all data sets), mean rank can reduce the susceptibility to outliers that, for instance, allows a classifier's excellent performance on one data set to compensate for its overall bad performance [69].

- Friedman test. As recommended by Demsar [69], the Friedman test is effective for comparing multiple algorithms across multiple data sets. It compares the mean ranks of schemes to decide whether to reject the null hypothesis, which states that all the schemes are equivalent and, so, their ranks should be equal.

- Nemenyi test. If the Friedman test rejects its null hypothesis, we can proceed with a post hoc test, the Nemenyi test. It can be applied to mean ranks of competing schemes and indicate whose performances have statistically significant differences (here, we use the 0.05 critical level).

- Win/lose/tie (w/1/t) record. This can be calculated for each pair of competitors $A$ and $B$ with regard to a performance measure $M$. The record represents the number of data sets in which $A$, respectively, beats, loses to, or ties with $B$ on $M$. To avoid breaking the flow of the main text, the $\mathrm{w} / 1 / \mathrm{t}$ records on error, bias, and variance are, respectively, listed in Tables 2, 3 , and 4 .

\subsection{Observations and Analyses}

Because information metrics can act as both selection and weighing schemes, we add a suffix " $s$ " to each selection scheme and " $w$ " to each weighing scheme. When acting as selection schemes, AIC, BIC, and MDL produce the same order of SPODEs and select the same ones. Hence, $\mathrm{MDL}_{s}$ represents the results for $\mathrm{AIC}_{s}$ and $\mathrm{BIC}_{s}$ as well. 
TABLE 2

Win/Lose/Tie Records of Each Pair of Competing Schemes on Reducing Classification Error

\begin{tabular}{|c|c|c|c|c|c|c|c|c|c|c|c|c|c|c|}
\hline $\begin{array}{l}\text { Error } \\
\mathrm{w} / \mathrm{l} / \mathrm{t}\end{array}$ & AODE & $\operatorname{RAN}_{s}$ & $\mathrm{MDL}_{s}$ & $\mathrm{MML}_{s}$ & $\mathrm{CV}_{s}$ & $\mathrm{FSA}_{s}$ & $\mathrm{BSE}_{s}$ & $\mathrm{LE}_{s}$ & $\mathrm{AIC}_{w}$ & $\mathrm{BIC}_{w}$ & $\mathrm{MDL}_{w}$ & $\mathrm{MML}_{w}$ & $\mathrm{BMA}_{w}$ & $\operatorname{MAPLMD}_{w}$ \\
\hline $\mathrm{RAN}_{s}$ & $11 / 29 / 18$ & & & & & & & & & & & & & \\
\hline $\mathrm{MDL}_{s}$ & $10 / 28 / 20$ & $16 / 16 / 26$ & & & & & & & & & & & & \\
\hline $\mathrm{MML}_{s}$ & $14 / 25 / 19$ & $23 / 16 / 19$ & $16 / 8 / 34$ & & & & & & & & & & & \\
\hline $\mathrm{CV}_{s}$ & $18 / 27 / 13$ & $18 / 19 / 21$ & $16 / 14 / 28$ & $13 / 17 / 28$ & & & & & & & & & & \\
\hline $\mathrm{FSA}_{s}$ & $20 / 29 / 9$ & $24 / 15 / 19$ & $25 / 17 / 16$ & $21 / 22 / 15$ & $23 / 17 / 18$ & & & & & & & & & \\
\hline $\mathrm{BSE}_{s}$ & $17 / 30 / 11$ & $24 / 17 / 17$ & $22 / 16 / 20$ & $19 / 20 / 19$ & $21 / 20 / 17$ & $17 / 16 / 25$ & & & & & & & & \\
\hline $\mathrm{LE}_{s}$ & $27 / 8 / 23$ & $40 / 5 / 13$ & $37 / 6 / 15$ & $36 / 6 / 16$ & $36 / 11 / 11$ & $40 / 13 / 5$ & $38 / 13 / 7$ & & & & & & & \\
\hline $\mathrm{AIC}_{w}$ & $6 / 48 / 4$ & $14 / 43 / 1$ & $11 / 45 / 2$ & $12 / 44 / 2$ & $12 / 43 / 3$ & $12 / 43 / 3$ & $12 / 43 / 3$ & $6 / 49 / 3$ & & & & & & \\
\hline $\mathrm{BIC}_{w}$ & $6 / 47 / 5$ & $13 / 43 / 2$ & $10 / 43 / 5$ & $12 / 43 / 3$ & $12 / 43 / 3$ & $13 / 42 / 3$ & $12 / 42 / 4$ & $6 / 48 / 4$ & $14 / 12 / 32$ & & & & & \\
\hline $\mathrm{MDL}_{w}$ & $6 / 48 / 4$ & $13 / 43 / 2$ & $8 / 45 / 5$ & $11 / 44 / 3$ & $11 / 44 / 3$ & $12 / 44 / 2$ & $11 / 43 / 4$ & $6 / 49 / 3$ & $9 / 10 / 39$ & $10 / 12 / 36$ & & & & \\
\hline $\mathrm{MML}_{w}$ & $8 / 11 / 39$ & $30 / 10 / 18$ & $27 / 10 / 21$ & $23 / 13 / 22$ & $25 / 18 / 15$ & $28 / 21 / 9$ & $29 / 20 / 9$ & $7 / 29 / 22$ & $49 / 7 / 2$ & $46 / 7 / 5$ & $48 / 7 / 3$ & & & \\
\hline $\mathrm{BMA}_{w}$ & $7 / 49 / 2$ & $9 / 46 / 3$ & $10 / 46 / 2$ & $9 / 47 / 2$ & $10 / 45 / 3$ & $9 / 47 / 2$ & $11 / 45 / 2$ & $4 / 52 / 2$ & $21 / 26 / 11$ & $24 / 25 / 9$ & $22 / 25 / 11$ & $7 / 49 / 2$ & & \\
\hline MAPLMD $_{w}$ & $25 / 15 / 18$ & $39 / 6 / 13$ & $39 / 6 / 13$ & $38 / 8 / 12$ & $34 / 13 / 11$ & $31 / 15 / 12$ & $35 / 14 / 9$ & $21 / 22 / 15$ & $48 / 7 / 3$ & 48/6/4 & $50 / 5 / 3$ & $27 / 13 / 18$ & $52 / 5 / 1$ & \\
\hline $\mathrm{MAPLMG}_{w}$ & $33 / 6 / 19$ & $47 / 2 / 9$ & $45 / 5 / 8$ & $44 / 4 / 10$ & $43 / 7 / 8$ & $39 / 7 / 12$ & $44 / 7 / 7$ & $28 / 19 / 11$ & $50 / 5 / 3$ & $49 / 6 / 3$ & $49 / 5 / 4$ & $36 / 6 / 16$ & $50 / 5 / 3$ & $26 / 10 / 22$ \\
\hline
\end{tabular}

TABLE 3

Win/Lose/Tie Records of Each Pair of Competing Schemes on Reducing Classification Bias

\begin{tabular}{|c|c|c|c|c|c|c|c|c|c|c|c|c|c|c|}
\hline $\begin{array}{l}\text { Bias } \\
\text { w/l/t }\end{array}$ & AODE & $\mathrm{RAN}_{s}$ & $\mathrm{MDL}_{s}$ & $\mathrm{MML}_{s}$ & $\mathrm{CV}_{s}$ & $\mathrm{FSA}_{s}$ & $\mathrm{BSE}_{s}$ & $\mathrm{LE}_{s}$ & $\mathrm{AIC}_{w}$ & $\mathrm{BIC}_{w}$ & $\mathrm{MDL}_{w}$ & $\mathrm{MML}_{w}$ & $\mathrm{BMA}_{w}$ & MAPLMD $_{w}$ \\
\hline $\mathrm{RAN}_{s}$ & $23 / 18 / 17$ & & & & & & & & & & & & & \\
\hline $\mathrm{MDL}_{s}$ & $20 / 20 / 18$ & $20 / 18 / 20$ & & & & & & & & & & & & \\
\hline $\mathrm{MML}_{s}$ & $28 / 17 / 13$ & 27/13/18 & $17 / 6 / 35$ & & & & & & & & & & & \\
\hline $\mathrm{CV}_{s}$ & $40 / 12 / 6$ & $40 / 6 / 12$ & $38 / 2 / 18$ & $36 / 8 / 14$ & & & & & & & & & & \\
\hline $\mathrm{FSA}_{s}$ & $43 / 11 / 4$ & $47 / 2 / 9$ & $41 / 3 / 14$ & $40 / 5 / 13$ & $20 / 14 / 24$ & & & & & & & & & \\
\hline $\mathrm{BSE}_{s}$ & $43 / 13 / 2$ & $45 / 5 / 8$ & $38 / 4 / 16$ & $32 / 9 / 17$ & $14 / 19 / 25$ & $5 / 23 / 30$ & & & & & & & & \\
\hline $\mathrm{LE}_{s}$ & $33 / 2 / 23$ & $35 / 12 / 11$ & $34 / 12 / 12$ & $30 / 15 / 13$ & $24 / 29 / 5$ & $22 / 31 / 5$ & $27 / 29 / 2$ & & & & & & & \\
\hline $\mathrm{AIC}_{w}$ & $16 / 33 / 9$ & $19 / 33 / 6$ & $17 / 35 / 6$ & $17 / 36 / 5$ & $14 / 40 / 4$ & $14 / 42 / 2$ & $16 / 40 / 2$ & $14 / 39 / 5$ & & & & & & \\
\hline $\mathrm{BIC}_{w}$ & $11 / 37 / 10$ & $16 / 36 / 6$ & $14 / 36 / 8$ & $15 / 37 / 6$ & $12 / 43 / 3$ & $13 / 43 / 2$ & $15 / 40 / 3$ & $11 / 40 / 7$ & $7 / 18 / 33$ & & & & & \\
\hline $\mathrm{MDL}_{w}$ & $15 / 33 / 10$ & $19 / 33 / 6$ & $16 / 35 / 7$ & $15 / 37 / 6$ & $13 / 41 / 4$ & $13 / 43 / 2$ & $14 / 41 / 3$ & $15 / 38 / 5$ & 7/10/41 & $13 / 7 / 38$ & & & & \\
\hline $\mathrm{MML}_{w}$ & $14 / 5 / 39$ & $20 / 19 / 19$ & $23 / 20 / 15$ & $18 / 28 / 12$ & $12 / 39 / 7$ & $11 / 43 / 4$ & $12 / 39 / 7$ & $4 / 33 / 21$ & $35 / 16 / 7$ & $38 / 11 / 9$ & $34 / 15 / 9$ & & & \\
\hline $\mathrm{BMA}_{w}$ & $19 / 31 / 8$ & $25 / 27 / 6$ & $25 / 27 / 6$ & $25 / 30 / 3$ & $18 / 36 / 4$ & $20 / 34 / 4$ & $21 / 33 / 4$ & $14 / 39 / 5$ & $27 / 21 / 10$ & $31 / 18 / 9$ & $27 / 20 / 11$ & $17 / 32 / 9$ & & \\
\hline MAPLMD $_{w}$ & $30 / 7 / 21$ & $37 / 13 / 8$ & $34 / 12 / 12$ & $29 / 18 / 11$ & $20 / 33 / 5$ & $15 / 36 / 7$ & $19 / 32 / 7$ & $21 / 24 / 13$ & $38 / 12 / 8$ & $41 / 11 / 6$ & $38 / 12 / 8$ & $31 / 11 / 16$ & $33 / 18 / 7$ & \\
\hline MAPLMG $_{w}$ & $36 / 2 / 20$ & $44 / 6 / 8$ & $41 / 10 / 7$ & $34 / 16 / 8$ & $22 / 26 / 10$ & $17 / 31 / 10$ & $22 / 24 / 12$ & $25 / 23 / 10$ & $41 / 10 / 7$ & $44 / 10 / 4$ & $43 / 10 / 5$ & $37 / 7 / 14$ & $37 / 15 / 6$ & $22 / 7 / 29$ \\
\hline
\end{tabular}

TABLE 4

Win/Lose/Tie Records of Each Pair of Competing Schemes on Reducing Classification Variance

\begin{tabular}{|c|c|c|c|c|c|c|c|c|c|c|c|c|c|c|}
\hline $\begin{array}{l}\text { Variance } \\
\mathrm{w} / 1 / \mathrm{t}\end{array}$ & AODE & $\operatorname{RAN}_{s}$ & $\mathrm{MDL}_{s}$ & $\mathrm{MML}_{s}$ & $\mathrm{CV}_{s}$ & $\mathrm{FSA}_{s}$ & $\mathrm{BSE}_{s}$ & $\mathrm{LE}_{s}$ & $\mathrm{AIC}_{w}$ & $\mathrm{BIC}_{w}$ & $\operatorname{MDL}_{w}$ & $\mathrm{MML}_{w}$ & $\mathrm{BMA}_{w}$ & MAPLMD $_{w}$ \\
\hline $\mathrm{RAN}_{s}$ & $12 / 35 / 11$ & & & & & & & & & & & & & \\
\hline $\mathrm{MDL}_{s}$ & $10 / 28 / 20$ & $16 / 19 / 23$ & & & & & & & & & & & & \\
\hline $\mathrm{MML}_{s}$ & 9/32/17 & $11 / 24 / 23$ & $7 / 18 / 33$ & & & & & & & & & & & \\
\hline $\mathrm{CV}_{s}$ & $9 / 44 / 5$ & $5 / 37 / 16$ & $2 / 33 / 23$ & $3 / 31 / 24$ & & & & & & & & & & \\
\hline $\mathrm{FSA}_{s}$ & $9 / 43 / 6$ & $3 / 38 / 17$ & $6 / 35 / 17$ & $9 / 29 / 20$ & $21 / 11 / 26$ & & & & & & & & & \\
\hline $\mathrm{BSE}_{s}$ & $8 / 44 / 6$ & $3 / 35 / 20$ & $8 / 32 / 18$ & $13 / 28 / 17$ & $27 / 16 / 15$ & $21 / 14 / 23$ & & & & & & & & \\
\hline $\mathrm{LE}_{s}$ & $8 / 21 / 29$ & $31 / 17 / 10$ & $26 / 16 / 16$ & $31 / 16 / 11$ & 43/9/6 & $42 / 11 / 5$ & $39 / 14 / 5$ & & & & & & & \\
\hline $\mathrm{AIC}_{w}$ & $10 / 40 / 8$ & $17 / 34 / 7$ & $16 / 33 / 9$ & $18 / 32 / 8$ & $24 / 27 / 7$ & $24 / 27 / 7$ & $24 / 28 / 6$ & $16 / 35 / 7$ & & & & & & \\
\hline $\mathrm{BIC}_{w}$ & $14 / 34 / 10$ & $18 / 32 / 8$ & $16 / 29 / 13$ & $18 / 30 / 10$ & $24 / 25 / 9$ & $26 / 24 / 8$ & $24 / 24 / 10$ & $18 / 33 / 7$ & $14 / 9 / 35$ & & & & & \\
\hline $\mathrm{MDL}_{w}$ & $11 / 39 / 8$ & $17 / 33 / 8$ & $16 / 32 / 10$ & $17 / 33 / 8$ & $26 / 26 / 6$ & $25 / 26 / 7$ & $23 / 27 / 8$ & $14 / 37 / 7$ & $8 / 9 / 41$ & $6 / 13 / 39$ & & & & \\
\hline $\mathrm{MML}_{w}$ & $8 / 11 / 39$ & $36 / 11 / 11$ & $29 / 10 / 19$ & $34 / 9 / 15$ & $44 / 8 / 6$ & $42 / 9 / 7$ & $45 / 8 / 5$ & $21 / 12 / 25$ & $43 / 10 / 5$ & $37 / 14 / 7$ & $41 / 11 / 6$ & & & \\
\hline $\mathrm{BMA}_{w}$ & $5 / 48 / 5$ & $8 / 45 / 5$ & $7 / 47 / 4$ & $8 / 47 / 3$ & $15 / 40 / 3$ & $13 / 41 / 4$ & $12 / 40 / 6$ & $6 / 47 / 5$ & $9 / 35 / 14$ & $11 / 37 / 10$ & $13 / 36 / 9$ & $5 / 47 / 6$ & & \\
\hline MAPLMD $_{w}$ & 9/27/22 & $27 / 15 / 16$ & $28 / 17 / 13$ & 29/15/14 & $41 / 8 / 9$ & $44 / 9 / 5$ & $42 / 8 / 8$ & $20 / 23 / 15$ & $38 / 11 / 9$ & $33 / 16 / 9$ & $37 / 13 / 8$ & $10 / 28 / 20$ & $47 / 6 / 5$ & \\
\hline MAPLMG $_{w}$ & $9 / 25 / 24$ & $27 / 12 / 19$ & $25 / 15 / 18$ & $31 / 13 / 14$ & $43 / 7 / 8$ & $45 / 7 / 6$ & $43 / 7 / 8$ & $22 / 20 / 16$ & $38 / 12 / 8$ & $34 / 15 / 9$ & $38 / 12 / 8$ & $10 / 25 / 23$ & $47 / 6 / 5$ & $14 / 15 / 29$ \\
\hline
\end{tabular}

\subsubsection{Reducing Classification Error}

Apply the Nemenyi test to alternative schemes' mean ranks of reducing error. To compare each scheme's influence on the SPODE ensemble's classification error, their mean ranks of reducing error are illustrated in Fig. 2. It indicates that among selection schemes, $\mathrm{LE}_{s}$ is the most effective on reducing classification error, whereas among weighing schemes, MAPLMG ${ }_{w}$ is the most effective. It also reveals an interesting point that AODE, which simply linearly combines every SPODE without any selection or weighing, is actually more effective than the majority of rival schemes.
We partially attribute this to AODE's outstanding performance on reducing variance, which will be discussed in Section 6.5.2.

When we apply the Friedman test, with 15 algorithms $^{2}$ and 58 data sets, $F_{F}$ is distributed according to the F distribution with $(15-1)=14$ and $(15-1) \times(58-1)=$ 798 degrees of freedom. The critical value of $F(14,798)$ at

2. We have studied 16 schemes. For experimental purpose, $\mathrm{AIC}_{s}$ and $\mathrm{BIC}_{s}$ are presented by $\mathrm{MDL}_{s}$ because they produce the same results. AODE is added as a benchmark algorithm. As a result, there are 15 algorithms tested. 


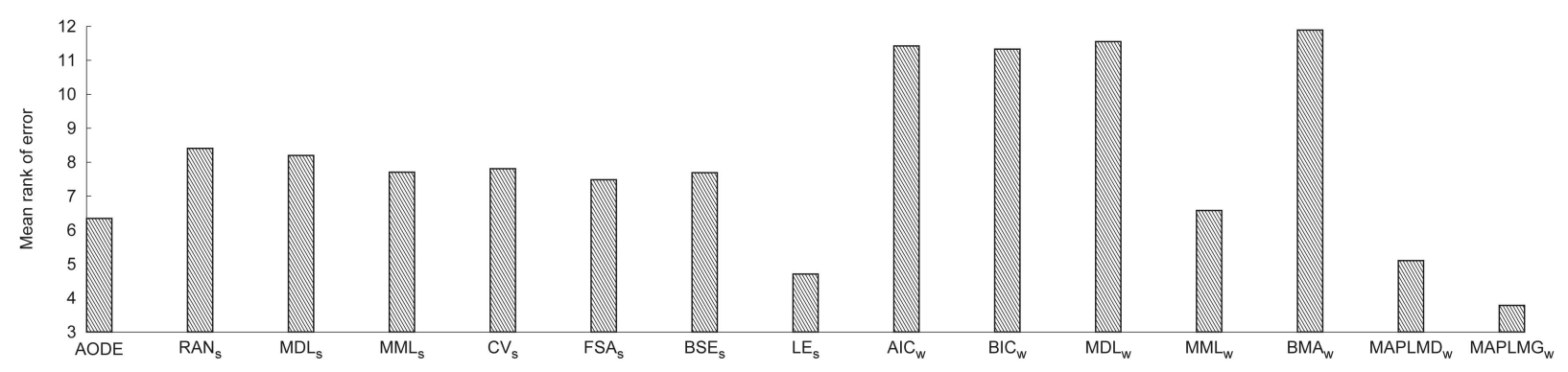

Fig. 2. Compare alternative methods' mean ranks of reducing error.

the 0.05 critical level is 1.7. $F_{F}$ calculated from the mean ranks is 28.3. Since $28.3>1.7$, we can reject the null hypothesis and infer that there exists a significant difference among rival schemes.

To find out exactly which schemes are significantly different, we proceed to the Nemenyi test, whose results are illustrated in Fig. 6. In the graph, the mean rank of each scheme is pointed by a circle. The horizontal bar across each circle indicates the "critical difference." The performance of two methods is significantly different if their corresponding mean ranks differ by at least the critical difference. That is, two methods are significantly different if their horizontal bars are not overlapping. For instance, Fig. 6 reveals that MAPLMG $_{w}$ is ranked best and is significantly better than $\mathrm{RAN}_{s}, \mathrm{MDL}_{s}, \mathrm{MML}_{s}, \mathrm{CV}_{s}, \mathrm{FSA}_{s}, \mathrm{BSE}_{s}, \mathrm{AIC}_{w}, \mathrm{BIC}_{w}, \mathrm{MDL}_{w}$, and $\mathrm{BMA}_{w}$.

\subsubsection{Reducing Classification Variance}

Fig. 4 illustrates each scheme's mean rank of reducing variance. The Friedman test indicates that there exist significant differences among schemes on reducing variance, and Fig. 7 depicts the results of the Nemenyi test to reveal what those differences are.

It is observed that $\mathrm{AODE}$ and $\mathrm{MML}_{w}$ are the best at reducing classification variance among alternative methods. Between themselves, AODE beats $\mathrm{MML}_{w}$ more often than not (w/1/t being 11/8/39) according to Table 4 . We suggest that the reason for AODE's outstanding performance on variance reduction is that selection and weighing will increase the classifier's sensitivity to training data because weights, as well as selection metrics, are calculated therefrom. In contrast, AODE minimizes dependence on training data and hence can minimize classification variance.

\subsubsection{Reducing Classification Bias}

Fig. 5 illustrates each scheme's mean rank of reducing bias. The Friedman test indicates that there exist significant

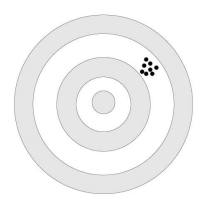

(a)

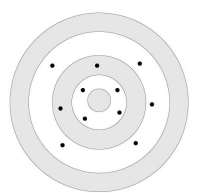

(b)

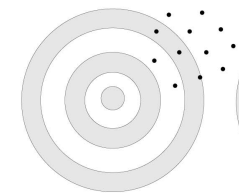

(c)

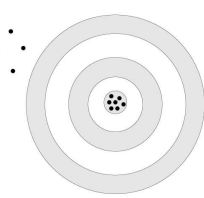

(d)
Fig. 3. Bias and variance in shooting arrows at a target. Bias means that the archer systematically misses the bull's-eye in the same direction. Variance means that the arrows are scattered [66]. (a) High bias, low variance. (b) Low bias, high variance. (c) High bias, high variance. (d) Low bias, low variance. differences among schemes on reducing bias, and Fig. 8 depicts the results of the Nemenyi test to reveal what those differences are.

It is observed that on reducing bias, model selection schemes like $\mathrm{FSA}_{s}, \mathrm{CV}_{s}$, and $\mathrm{BSE}_{s}$ are the most effective. However, their outstanding capability for bias reduction is overshadowed by their inferior performance on variance reduction (refer to Fig. 7). The net effect is that they are worse at reducing error for SPODE ensembles. In contrast, schemes like $\mathrm{LE}_{s}$ and MAPLMG ${ }_{w}$ reduce bias, as well as control variance, and turn out to be more effective at error reduction for SPODE ensembles.

\subsubsection{Capability for Fast Training}

Fig. 9 illustrates the mean ranks of alternative schemes' training time. Consistent with our time complexity analyses in Section 5.1, $\mathrm{AODE}$ and $\mathrm{LE}_{s}$, which do not conduct any selection or weighing work in training, are the most efficient. $\mathrm{MAPLMD}_{w}$ and $\mathrm{MAPLMG}_{w}$ optimize multiple weights simultaneously, which very likely contributes to their effectiveness since others calculate the weights for individual SPODEs in isolation. On the other hand, this optimization demands time, and hence, $\mathrm{MAPLMD}_{w}$ and $\mathrm{MAPLMG}_{w}$ are slower than every other scheme except $\mathrm{MML}_{w}$ and $\mathrm{BMA}_{w}$.

MML and BMA can return large values. In that case, when serving as weighing schemes, $\mathrm{MML}_{w}$ and $\mathrm{BMA}_{w}$ involve calculating large exponentials in (11). This often leads to arithmetic overflow when using 32-bit computing machines. Our solution to this problem is to use the java class BigDecimal that implements arbitrary-precision signed decimal numbers. A BigDecimal consists of an arbitraryprecision integer unscaled value and a nonnegative 32-bit integer scale, which represents the number of digits to the right of the decimal point. Although BigDecimal solves the problem of overflowing, its calculation can be very slow when the numbers are large. This is why $\mathrm{MML}_{w}$ and $\mathrm{BMA}_{w}$ are ranked the worst in Fig. 9 and require a large amount of training time, as in Fig. 10.

Hence, although $\mathrm{MML}_{w}$ is as effective as AODE in reducing classification variance and is ranked fifth in reducing classification error, it can be infeasible for modern real-world applications where large data sets are commonly involved.

\subsubsection{Capability for Fast Classification}

Figs. 11 and 12 illustrate alternative schemes' training time. Consistent with our time complexity analyses in Section 5.2, 


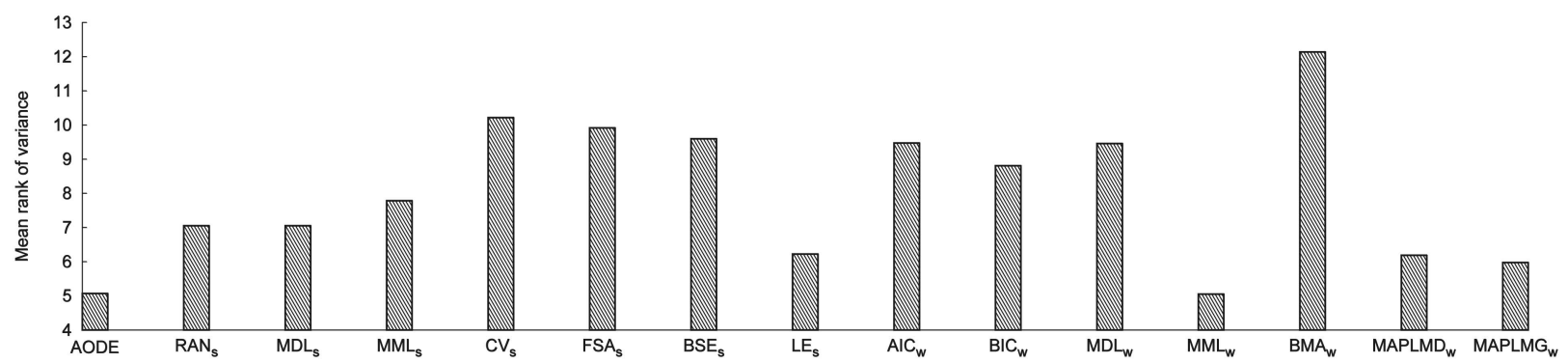

Fig. 4. Compare alternative methods' mean ranks of reducing variance.

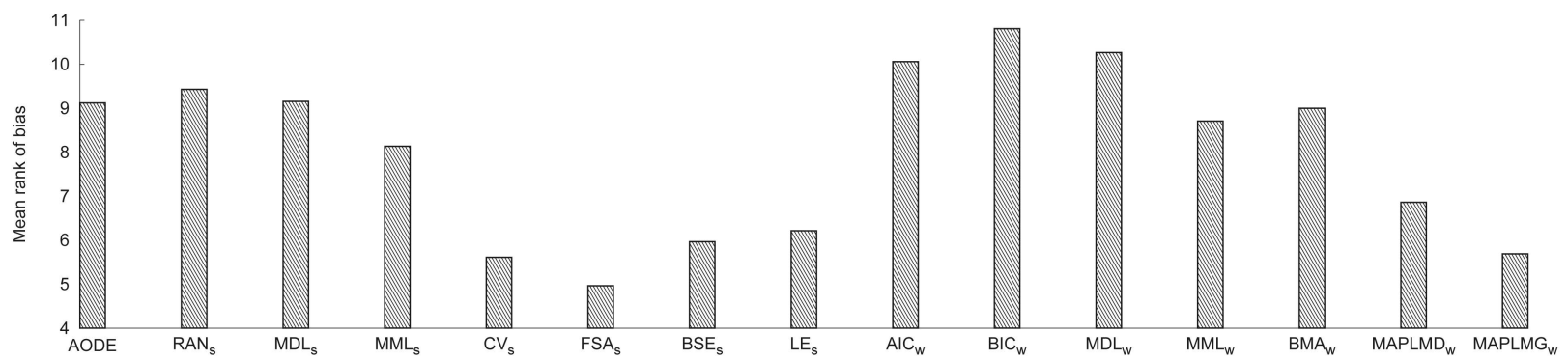

Fig. 5. Compare alternative methods' mean ranks of reducing bias.

model selection schemes identify a subset of SPODEs to carry out classification and hence are faster than AODE, which uses all SPODEs. Model weighing schemes uses all SPODEs and multiply each with calculated weights and hence are slower than AODE. Among all schemes, $\mathrm{FSA}_{s}$ delivers the fastest classification and $\mathrm{CV}_{s}$ the second.

One very interesting issue to spot is that $\mathrm{LE}_{s}$, a lazy method that conducts calculation in classification time, turns out to be faster than AODE. We suggest that the reason is that on one hand, $\mathrm{LE}_{s}$ requires a simple test each time when a pair of attribute values is considered to determine whether one is a generalization of the other, which causes a small increase in the computation time. On the other hand, once a generalization relationship is detected, $\mathrm{LE}_{s}$ need not calculate or multiply related conditional probabilities, which decreases the computation time. The time saved in the latter often exceeds the time cost

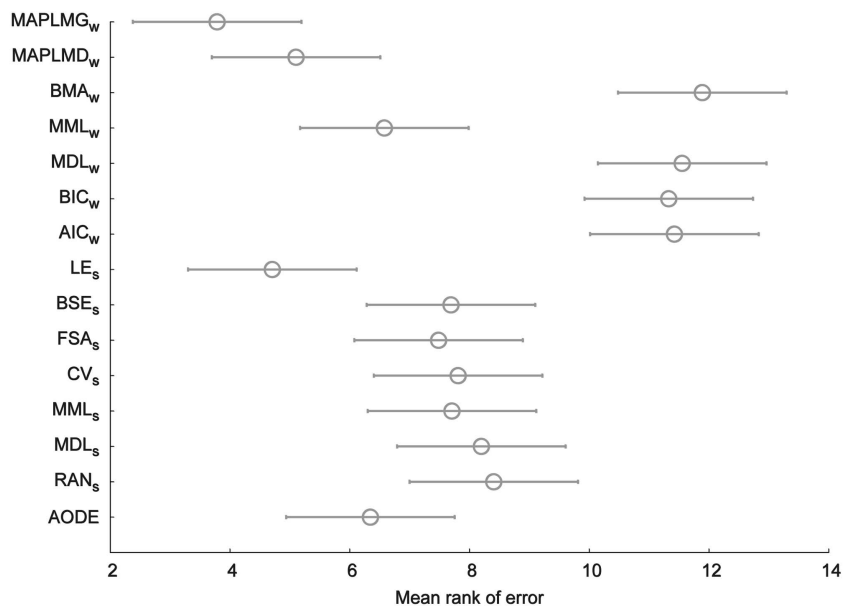

Fig. 6. Apply the Nemenyi test to alternative schemes' mean ranks of reducing error. in the former. Hence, $\mathrm{LE}_{s}$, although "lazy," can still deliver faster classification than AODE.

\subsubsection{Best Schemes' Relative Performance}

$\mathrm{LE}_{s}$ and $\mathrm{MAPLMG}_{w}$ are, respectively, the best model selection and model weighing schemes for reducing SPODE ensembles' classification error. Fig. 13 graphs the relative bias, variance, and error between $\mathrm{LE}_{s}, \mathrm{MAPLMG}_{w}$, and AODE. The values on the $y$-axis are the outcome for $\mathrm{LE}_{s}$ divided by that for AODE. The values of the $x$-axis are the outcome for MAPLMG ${ }_{w}$ divided by that for AODE. Each point on the graph represents one of the 58 data sets. Points on the left of the vertical line at $\mathrm{MAPLMG}_{w} / \mathrm{AODE}=1 \mathrm{in}$ each subgraph are those of which $\mathrm{MAPLMG}_{w}$ outperforms AODE. Points below the horizontal line at $\mathrm{LE}_{s} / \mathrm{AODE}=1$ indicate that $\mathrm{LE}_{s}$ outperforms AODE. Points below the

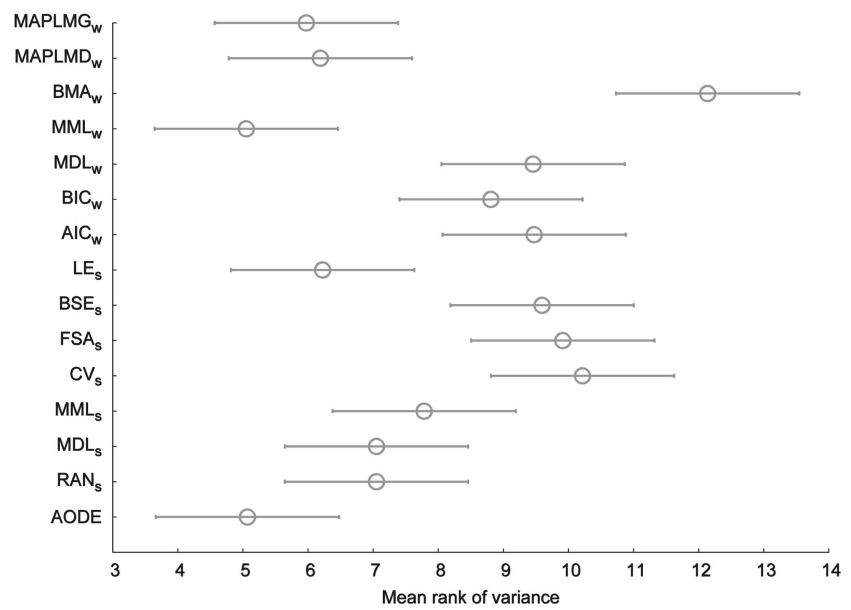

Fig. 7. Apply the Nemenyi test to alternative schemes' mean ranks of reducing variance. 


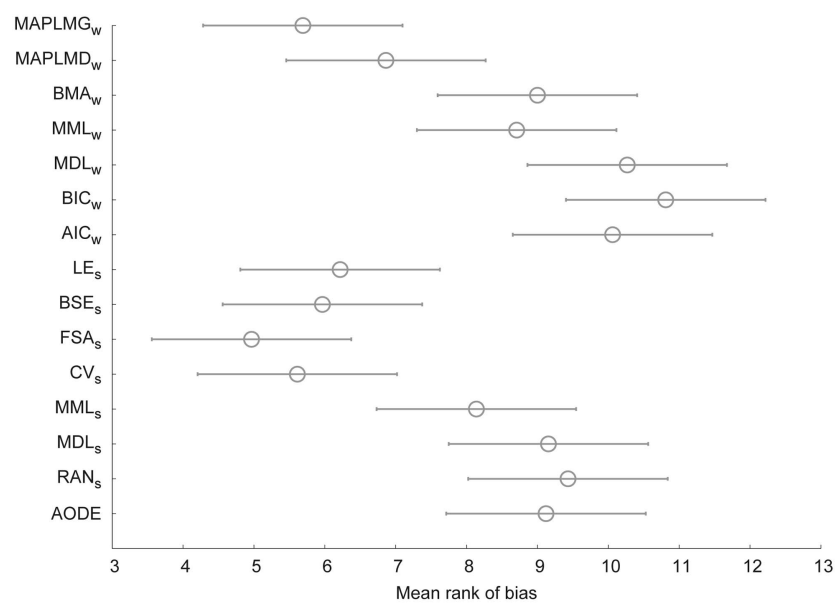

Fig. 8. Apply the Nemenyi test to alternative schemes' mean ranks of reducing bias.

diagonal line $\mathrm{Y}=\mathrm{X}$ represent that $\mathrm{MAPLMG}_{w}$ outperforms $\mathrm{LE}_{s}$. It is observed that on one hand, both $\mathrm{LE}_{s}$ and $\mathrm{MAPLMG}_{w}$ frequently reduce bias compared with AODE as the majority of points fall within the boundaries $\mathrm{X}=1$ and $Y=1$ in Fig. 13a. On the other hand, AODE is better at reducing variance as the majority of points fall beyond the boundaries $\mathrm{X}=1$ and $\mathrm{Y}=1$ in Fig. 13b. The end effect is that both $\mathrm{LE}_{s}$ and $\mathrm{MAPLMG}_{w}$ outperform AODE on reducing error $(\mathrm{w} / 1 / \mathrm{t}$ being $27 / 8 / 23$ and $33 / 6 / 19$, respectively, as in Table 2).

Between $\mathrm{LE}_{s}$ and MAPLMG ${ }_{w}$ themselves, it is observed that $\mathrm{MAPLMG}_{w}$ slightly outperforms $\mathrm{LE}_{s}$ on both bias and variance reduction (w/1/t being 25/23/10 and 22/20/16, respectively, as in Tables 3 and 4 ). The end effect is that

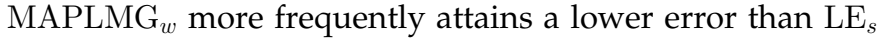

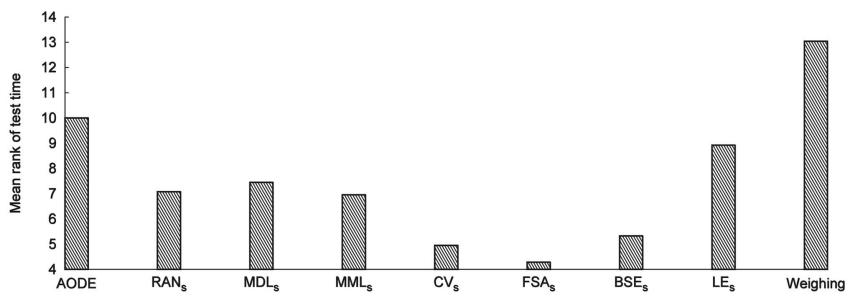

Fig. 11. Compare alternative schemes' mean ranks of test time.

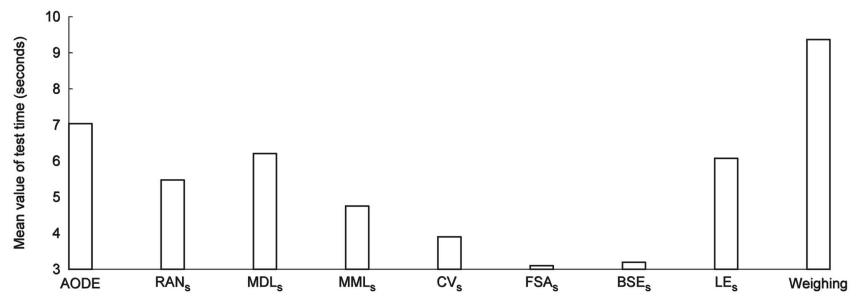

Fig. 12. Compare alternative schemes' mean values of test time.

(w/l/t being 28/19/11, as in Table 2). On the other hand, in terms of training efficiency and classification efficiency, $\mathrm{LE}_{s}$ is much faster than MAPLMG ${ }_{w}$, as detailed in Sections 6.5.4 and 6.5.5.

\section{Conclusion and Future Work}

This paper presents a comprehensive study, both theoretically and empirically, of 16 representative model selection and model weighing schemes for linearly ensembling SPODEs, a popular family of seminaive Bayesian classifiers.

For each scheme, this paper provides its definition, rationale, and time complexity. Comprehensive experiments across $58 \mathrm{UCI}$ benchmark data sets are conducted

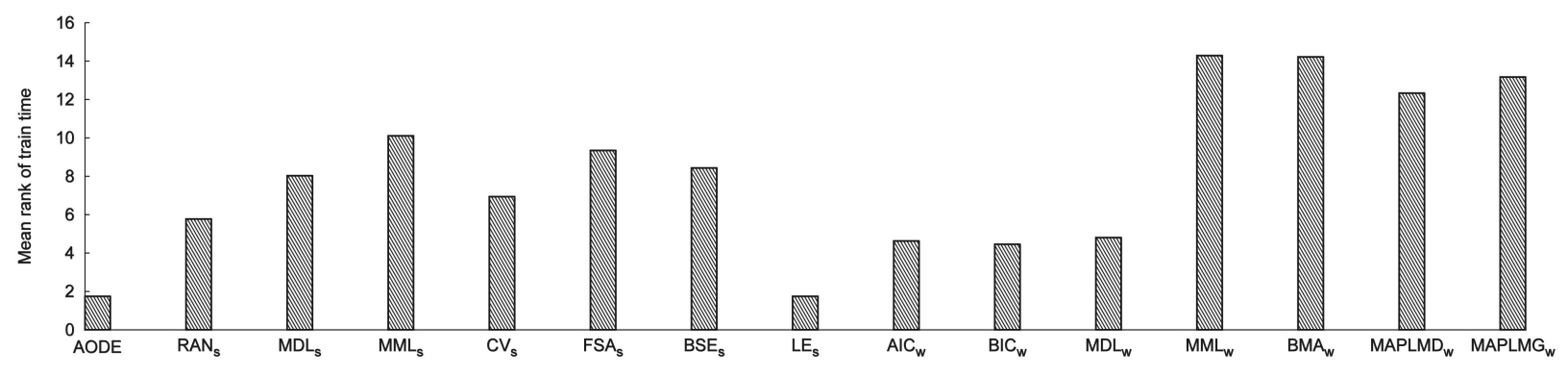

Fig. 9. Compare alternative schemes' mean ranks of training time.

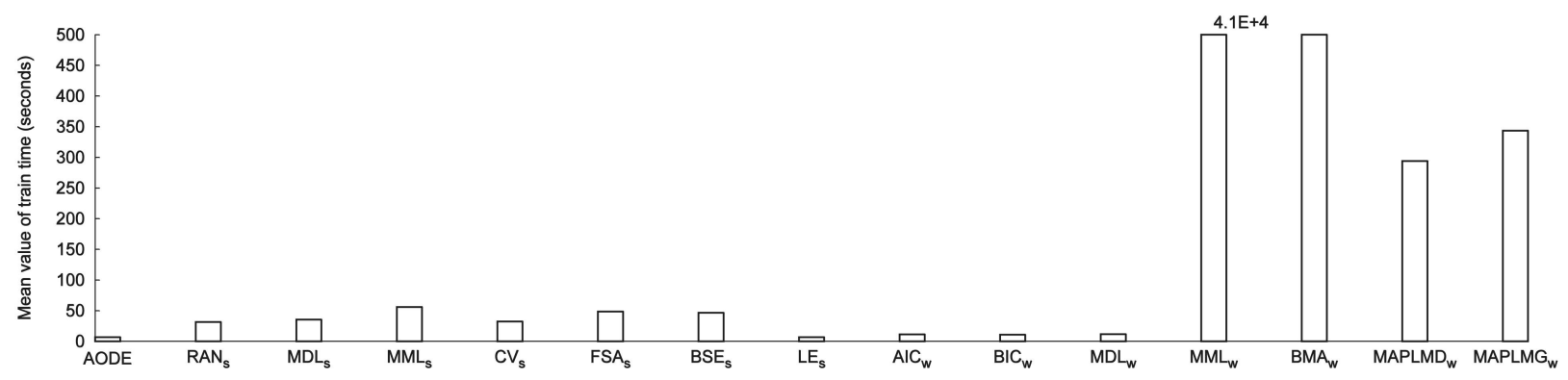

Fig. 10. Compare alternative schemes' mean values of training time. $\mathrm{MML}_{w}$ and $\mathrm{BMA}_{w}$ suffer from the overflowing problem in practice. To keep a readable scale, the bars of $\mathrm{MML}_{w}$ and $\mathrm{BMA}_{w}$ are cut short with their true values labeled on top. 


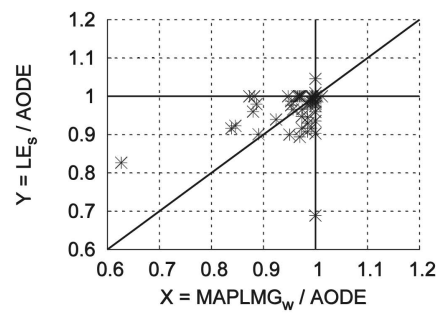

(a)

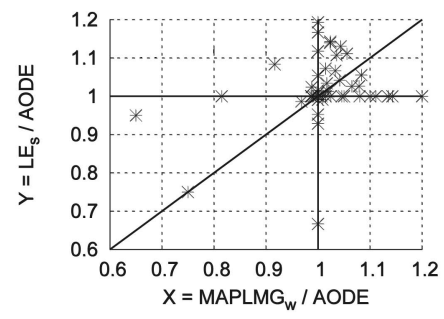

(b)

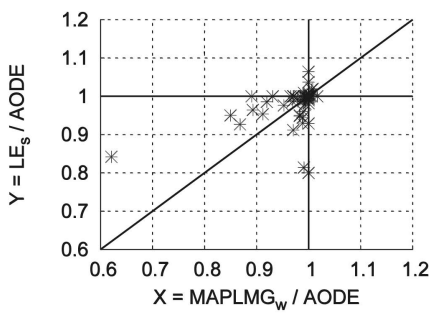

(c)

Fig. 13. Illustrate $\mathrm{LE}_{s}$ and $\mathrm{MAPLMG}_{w}$ 's performance relative to AODE.

to test each scheme's effect on ensemble learning's accuracy and efficiency.

The study results suggest the following answers to the questions we have asked at the beginning of the paper:

1. $\mathrm{MAPLMG}_{w}$ is ranked the best among all rival schemes on classification accuracy. It wins more often than not when compared with every other single scheme across the suite of 58 data sets. It is significantly better than $\mathrm{RAN}_{s}, \mathrm{MDL}_{s}, \mathrm{MML}_{s}, \mathrm{CV}_{s}$, $\mathrm{FSA}_{s}, \mathrm{BSE}_{s}, \mathrm{AIC}_{w}, \mathrm{BIC}_{w}, \mathrm{MDL}_{w}$, and $\mathrm{BMA}_{w}$. However, its training takes longer than the majority of the schemes.

2. $\mathrm{LE}_{s}$ is ranked the best among model selection schemes and the second best among all rival schemes on classification accuracy. It wins more often than not when compared with every other single scheme except MAPLMG ${ }_{w}$ across the suite of 58 data sets. It is significantly better than $\mathrm{RAN}_{s}$, $\mathrm{MDL}_{s}, \mathrm{MML}_{s}, \mathrm{CV}_{s}, \mathrm{BSE}_{s}, \mathrm{AIC}_{w}, \mathrm{BIC}_{w}, \mathrm{MDL}_{w}$, and $\mathrm{BMA}_{w}$. Besides, it is the most efficient at training, as well as very efficient at classification.

3. AODE is ranked the best among all rival methods on reducing classification variance. It wins more often than not compared with every other single scheme across the suite of 58 data sets. It is significantly better than $\mathrm{CV}_{s}, \mathrm{FSA}_{s}, \mathrm{BSE}_{s}, \mathrm{AIC}_{w}, \mathrm{BIC}_{w}, \mathrm{MDL}_{w}$, and $\mathrm{BMA}_{w}$. It is the most efficient at training. It is faster than weighing schemes and slower than selection schemes at classification.

4. Commonly used selection schemes such as $\mathrm{CV}_{s}$, $\mathrm{FSA}_{s}$, and $\mathrm{BSE}_{s}$ turn out to be less effective than simply including every candidate classifier (AODE). The reason is that they incur high classification variance. Although they are ranked among the best on reducing classification bias, their wins in bias reduction are overshadowed by their losses in variance reduction. The end effect is that they are less effective at reducing error on the learning tasks investigated.

5. The observation that MAPLMD ${ }_{w}$ is less effective than $\mathrm{MAPLMG}_{w}$ suggests that combining joint (generic) probabilities $P_{i}(y, \mathbf{x})$ leads to more accurate classification than combining conditional (discriminative) probabilities $P_{i}(y \mid \mathbf{x})$. In practice, $P_{i}(y, \mathbf{x})$ is estimated from $\operatorname{count}(y, \mathbf{x})$, the count of training instances $\langle\mathbf{x}, y\rangle$, whereas $P_{i}(y \mid \mathbf{x})$ is estimated from the count of training instances $\mathrm{x}$ in addition to $\operatorname{count}(y, \mathbf{x})$. Hence, it is suggested that estimating
$P_{i}(y \mid \mathbf{x})$ is less reliable than estimating $P_{i}(y, \mathbf{x})$ and is not preferred.

6. In general, information-theoretic metrics (either as selection or as weighing schemes) are not effective at reducing an ensemble's classification error. Although $\mathrm{MML}_{w}$ as a weighing scheme is ranked fairly well (fifth), its high time requirement for calculating weights hinders its deployment in practice. A further thought is that currently, information-theoretic metrics measure the merit of individual classifiers. It might help to generalize them so as to measure the collective merit of an ensemble. This can be an interesting research issue to further explore.

7. Hence, whether to use model selection or model weighing depends on the specific requirements of a particular classification task. If one needs to maximize accuracy, we recommend MAPLMG ${ }_{w}$. If one seeks both high learning accuracy and efficiency, we recommend $\mathrm{LE}_{s}$. If one needs to minimize variance while obtaining a reasonable accuracy, we recommend AODE.

The model selection and weighing schemes studied here can be generalized to other Bayesian network classifiers. RAN, CV, FSA, and BSE only utilize a classifier's classifications. Hence, they can be applied to any classifier. LE seeks generalization relationships among attribute values. If such a relationship exists, it deletes the more general value from the network structure. The calculations for AIC and BIC, MDL, MML, and BMA with MML have already been extended to arbitrary Bayesian network structures [70], [71], [51], [72]. MAPLMG and MAPLMD were introduced as linear mixture inducers for either generative or discriminative probabilistic classifiers [31] and are then particularized to SPODE in this study. Hence, they can be directly applied to any Bayesian network classifier. An interesting direction for future research is to examine the extent to which our results generalize to other Bayesian network classifiers.

\section{ACKNOWLEDGMENTS}

The authors gratefully acknowledge Dr. Janez Demsar for his kind help on their statistical tests and Dr. Gavin Brown for his kind help on the collection of ensemble learning publications. This research was supported by Australian Research Council Grant DP0556279.

\section{REFERENCES}

[1] G. Brown, "The Ensemble Learning Bibliography," http:// www.cs.man.ac.uk/ gbrown/ensemblebib/, 2006. 
[2] A.C. Achilles, "The Collection of Computer Science Bibliographies," http://liinwww.ira.uka.de/bibliography/Neural/ EnsembleLearning.html, 2006.

[3] R. Caruana, A. Niculescu, G. Crew, and A. Ksikes, "Ensemble Selection from Libraries of Models," Proc. 21st Int'l Conf. Machine Learning, 2004.

[4] L. Kuncheva, "Switching between Selection and Fusion in Combining Classifiers: An Experiment," IEEE Trans. Systems, Man, and Cybernetics, vol. 32, no. 2, pp. 146-156, 2002.

[5] R.E. Banfield, L.O. Hall, K.W. Bowyer, and W.P. Kegelmeyer, "A Comparison of Decision Tree Ensemble Creation Techniques," IEEE Trans. Pattern Analysis and Machine Learning, vol. 29, no. 1, pp. 173-180, Jan. 2007.

[6] E.J. Keogh and M.J. Pazzani, "Learning Augmented Bayesian Classifiers: A Comparison of Distribution-Based and Classification-Based Approaches," Proc. Int'l Workshop Artificial Intelligence and Statistics, pp. 225-230, 1999.

[7] E.J. Keogh and M.J. Pazzani, "Learning the Structure of Augmented Bayesian Classifiers," Int'l J. Artificial Intelligence Tools, vol. 11, no. 40, pp. 587-601, 2002.

[8] B. Cestnik, I. Kononenko, and I. Bratko, "Assistant 86: A Knowledge-Elicitation Tool for Sophisticated Users," Proc. Second European Working Session on Learning (ESWL '87), pp. 31-45, 1987.

[9] P. Clark and T. Niblett, "The CN2 Induction Algorithm," Machine Learning, vol. 3, pp. 261-283, 1989.

[10] B. Cestnik, "Estimating Probabilities: A Crucial Task in Machine Learning," Proc. Ninth European Conf. Artificial Intelligence (ECAI '90), pp. 147-149, 1990.

[11] I. Kononenko, "Comparison of Inductive and Naive Bayesian Learning Approaches to Automatic Knowledge Acquisition," Current Trends in Knowledge Acquisition, chapter, IOS Press, 1990.

[12] P. Langley, W. Iba, and K. Thompson, "An Analysis of Bayesian Classifiers," Proc. 10th Nat'l Conf. Artificial Intelligence (AAAI '92), pp. 223-228, 1992.

[13] P. Domingos and M.J. Pazzani, "Beyond Independence: Conditions for the Optimality of the Simple Bayesian Classifier," Proc. 13th Int'l Conf. Machine Learning (ICML '96), pp. 105-112, 1996.

[14] P. Domingos and M.J. Pazzani, "On the Optimality of the Simple Bayesian Classifier under Zero-One Loss," Machine Learning, vol. 29, pp. 103-130, 1997.

[15] H. Zhang, C.X. Ling, and Z. Zhao, "The Learnability of Naive Bayes," Proc. Canadian Artificial Intelligence Conf., pp. 432-441, 2000.

[16] N. Friedman, D. Geiger, and M. Goldszmidt, "Bayesian Network Classifiers," Machine Learning, vol. 29, no. 2, pp. 131-163, 1997.

[17] J. Kittler, "Feature Selection and Extraction," Handbook of Pattern Recognition and Image Processing, T.Y. Young and K.S. Fu, eds., 1986.

[18] R. Kohavi, "Scaling Up the Accuracy of Naive-Bayes Classifiers: A Decision-Tree Hybrid," Proc. Second ACM SIGKDD Int'l Conf. Knowledge Discovery and Data Mining (KDD '96), pp. 202-207, 1996.

[19] I. Kononenko, "Semi-Naive Bayesian Classifier," Proc. European Working Session on Machine Learning (EWSL '91), pp. 206-219, 1991.

[20] P. Langley, "Induction of Recursive Bayesian Classifiers," Proc. European Conf. Machine Learning (ECML '93), pp. 153-164, 1993.

[21] P. Langley and S. Sage, "Induction of Selective Bayesian Classifiers," Proc. 10th Ann. Conf. Uncertainty in Artificial Intelligence (UAI '94), pp. 399-406, 1994.

[22] M.J. Pazzani, "Constructive Induction of Cartesian Product Attributes," ISIS: Information, Statistics and Induction in Science, pp. 66-77, 1996.

[23] M. Sahami, "Learning Limited Dependence Bayesian Classifiers," Proc. Second ACM SIGKDD Int'l Conf. Knowledge Discovery and Data Mining (KDD '96), pp. 334-338, 1996.

[24] M. Singh and G.M. Provan, "Efficient Learning of Selective Bayesian Network Classifiers," Proc. 13th Int'l Conf. Machine Learning (ICML '96), pp. 453-461, 1996.

[25] Z. Xie, W. Hsu, Z. Liu, and M.L. Lee, "SNNB: A Selective Neighborhood Based Naive Bayes for Lazy Learning," Proc. Sixth Pacific-Asia Conf. Advances in Knowledge Discovery and Data Mining (PAKDD '02), pp. 104-114, 2002.

[26] Z. Zheng and G.I. Webb, "Lazy Learning of Bayesian Rules," Machine Learning, vol. 41, no. 1, pp. 53-84, 2000.

[27] Z. Zheng, G.I. Webb, and K.M. Ting, "Lazy Bayesian Rules: A Lazy Semi-Naive Bayesian Learning Technique Competitive to Boosting Decision Trees," Proc. 16th Int'l Conf. Machine Learning (ICML '99), pp. 493-502, 1999.
[28] G.I. Webb, “Candidate Elimination Criteria for Lazy Bayesian Rules," Proc. 14th Australian Joint Conf. Artificial Intelligence, pp. 545-556, 2001

[29] G.I. Webb and M.J. Pazzani, "Adjusted Probability Naive Bayesian Induction," Proc. 11th Australian Joint Conf. Artificial Intelligence, pp. 285-295, 1998.

[30] G.I. Webb, J. Boughton, and Z. Wang, "Not So Naive Bayes: Aggregating One-Dependence Estimators," Machine Learning, vol. 58, no. 1, pp. 5-24, Jan. 2005.

[31] J. Cerquides and R.L. de Mántaras, "Robust Bayesian Linear Classifier Ensembles," Proc. 16th European Conf. Machine Learning (ECML '05), pp. 72-83, 2005.

[32] L. De Ferrari, "Mining Housekeeping Genes with a Naive Bayes Classifier," MSc thesis, School of Informatics, Univ. of Edinburgh, 2005.

[33] K. Flikka, L. Martens, J. Vandekerckhove, K. Gevaert, and I. Eidhammeri, "Improving Throughput and Reliability of Peptide Identifications through Spectrum Quality Evaluation," Proc. Ninth Ann. Int'l Conf. Research in Computational Molecular Biology (RECOMB '05), 2005.

[34] A.P. Nikora, "Classifying Requirements: Towards a More Rigorous Analysis of Natural-Language Specifications," Proc. 16th IEEE Int'l Symp. Software Reliability Eng. (ISSRE '05), pp. 291-300, 2005.

[35] Y. Yang, K. Korb, K.M. Ting, and G.I. Webb, "Ensemble Selection for Superparent-One-Dependence Estimators," Proc. 18th Australian Joint Conf. Artificial Intelligence, 2005.

[36] F. Zheng and G.I. Webb, "A Comparative Study of Semi-Naive Bayes Methods in Classification Learning," Proc. Fourth Australasian Data Mining Conf. (AusDM '05), pp. 141-156, 2005.

[37] S.B. Kotsiantis and P.E. Pintelas, "Logitboost of Simple Bayesian Classifier," Informatica, vol. 29, pp. 53-59, 2005.

[38] H. Zhang, L. Jiang, and J. Su, "Hidden Naive Bayes," Proc. 20th Nat'l Conf. Artificial Intelligence (AAAI '05), pp. 919-924, 2005.

[39] F. Birzele and S. Kramer, "A New Representation for Protein Secondary Structure Prediction Based on Frequent Patterns," Bioinformatics, vol. 22, no. 21, pp. 2628-2634, 2006.

[40] J. Su and H. Zhang, "Representing Conditional Independence Using Decision Trees," Proc. 20th Nat'l Conf. Artificial Intelligence (AAAI '05)), pp. 874-879, 2005.

[41] H. Zhang, L. Jiang, and J. Su, "Augmenting Naive Bayes for Ranking," Proc. 22nd Int'l Conf. Machine Learning (ICML '05), pp. 1020-1027, 2005.

[42] J. Abellan, "Application of Uncertainty Measures on Credal Sets on the Naive Bayesian Classifier," Int'l J. General Systems, vol. 35, no. 6, pp. 675-686, 2006.

[43] H. Yin, G. Li, T.Y. Leong, V. Kuralmani, H. Pang, B.T. Ang, K.K. Lee, and I. Ng, "Experimental Analysis on Severe Head Injury Outcome Prediction-A Preliminary Study," Technical Report TRD9/06, School of Computing, Nat'l Univ. of Singapore.

[44] F. Zheng and G.I. Webb, "Efficient Lazy Elimination for Averaged One-Dependence Estimators," Proc. 23rd Int'l Conf. Machine Learning (ICML '06), pp. 1113-1120, 2006.

[45] D. Zeng, S. Zhang, Z. Cai, S. Jiang, and L. Jiang, "A Novel Onedependence Estimator Based on Multi-Parents," Proc. Sixth Int'l Conf. Intelligent Systems Design and Applications (ISDA '06), pp. 639643, 2006.

[46] M. Ceci, "Naive Bayesian Learning from Structural Data," PhD dissertation, Dipartimento di Informatica, Univ. of Bari, Italy, 2005.

[47] Y. Yang, G. Webb, J. Cerquides, K. Korb, J. Boughton, and K.M. Ting, "To Select or to Weigh: A Comparative Study of Model Selection and Model Weighing for SPODE Ensembles," Proc. 17th European Conf. Machine Learning (ECML '06), pp. 533-544, 2006.

[48] H. Akaike, "A New Look at the Statistical Model Identification," IEEE Trans. Automatic Control, vol. 19, pp. 716-723, 1974.

[49] G. Schwarz, "Estimating the Dimension of a Model," Annals of Statistics, vol. 6, pp. 461-465, 1978.

[50] J. Suzuki, "Learning Bayesian Belief Networks Based on the MDL Principle: An Efficient Algorithm Using the Branch and Bound Technique," Proc. 13th Int'l Conf. Machine Learning (ICML '96), pp. 463-470, 1996.

[51] K. Korb and A. Nicholson, Bayesian Artificial Intelligence. Chapman \& Hall/CRC, 2004.

[52] G.F. Cooper and E. Herskovits, "A Bayesian Method for the Induction of Probabilistic Networks from Data," Machine Learning, vol. 9, pp. 309-347, 1992. 
[53] C.E. Shannon, "A Mathematical Theory of Communication," Bell System Technical J., vol. 27, no. 3, pp. 379-423, 1948.

[54] J.A. Hoeting, D. Madigan, A.E. Raftery, and C.T. Volinsky, "Bayesian Model Averaging: A Tutorial," Statistical Science, vol. 14, no. 4, pp. 382-417, 1999.

[55] P. Domingos, "Bayesian Averaging of Classifiers and the Overfitting Problem," Proc. 17th Int'l Conf. Machine Learning (ICML '00), pp. 223-230, 2000.

[56] P. Pedregal, "Introduction to Optimization," Texts in Applied Math., Springer, vol. 46, 2004.

[57] M.T. Heath, Scientific Computing: An Introductory Survey, second ed. McGraw-Hill, 2002.

[58] G. McLachlan and T. Krishnan, The EM Algorithm and Extensions. Wiley-Interscience, 1997.

[59] I.H. Witten and E. Frank, Data Mining: Practical Machine Learning Tools and Techniques with Java Implementations, second ed. Morgan Kaufmann, 2005.

[60] C.L. Blake and C.J. Merz, "UCI Repository of Machine Learning Databases," Dept. of Information and Computer Science, Univ. of California, Irvine, http://www.ics.uci.edu/ $\sim$ mlearn/mlrepository.html, 1998.

[61] G.I. Webb, "Multiboosting: A Technique for Combining Boosting and Wagging," Machine Learning, vol. 40, no. 2, pp. 159-196, 2000.

[62] L. Breiman, "Bias, Variance and Arcing Classifiers," Technical Report 460, Statistics Dept., Univ. of California, Berkeley, 1996.

[63] J.H. Friedman, "On Bias, Variance, 0/1-Loss, and the Curse-ofDimensionality," Data Mining and Knowledge Discovery, vol. 1, no. 1, pp. 55-77, 1997.

[64] R. Kohavi and D. Wolpert, "Bias Plus Variance Decomposition for Zero-One Loss Functions," Proc. 13th Int'l Conf. Machine Learning (ICML '96), pp. 275-283, 1996.

[65] E.B. Kong and T.G. Dietterich, "Error-Correcting Output Coding Corrects Bias and Variance," Proc. 12th Int'l Conf. Machine Learning (ICML '95), pp. 313-321, 1995.

[66] D.S. Moore and G.P. McCabe, Introduction to the Practice of Statistics, fourth ed. Michelle Julet, 2002.

[67] M. Friedman, "The Use of Ranks to Avoid the Assumption of Normality Implicit in the Analysis of Variance," J. Am. Statistical Assoc., vol. 32, pp. 675-701, 1937.

[68] M. Friedman, "A Comparison of Alternative Tests of Significance for the Problem of m Rankings," Annals of Math. Statistics, vol. 11, pp. 86-92, 1940.

[69] J. Demsar, "Statistical Comparisons of Classifiers over Multiple Data Sets," J. Machine Learning Research, vol. 7, pp. 1-30, 2006.

[70] T. Silander and P. Myllymaki, "A Simple Approach for Finding the Globally Optimal Bayesian Network Structure," Proc. 22nd Ann. Conf. Uncertainty in Artificial Intelligence (UAI '06), 2006.

[71] N. Friedman and M. Goldszmidt, "Learning Bayesian Networks with Local Structure," Proc. 12th Ann. Conf. Uncertainty in Artificial Intelligence, pp. 252-262, 1996.

[72] R. O'Donnell, "Learning Flexible Causal Models with MML," PhD dissertation, Monash Univ., 2007.

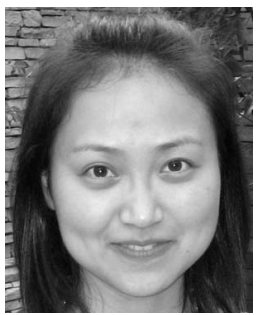

Ying Yang received the $\mathrm{PhD}$ degree in computer science from Monash University, Australia, in 2003. Following academic appointments at the University of Vermont, she is currently a research fellow at Monash University. She is recognized for her contributions in the fields of machine learning and data mining. She has published many scientific papers and book chapters on Bayesian classification learning, data stream mining, anytime learning, noise cleansing, and discretization.

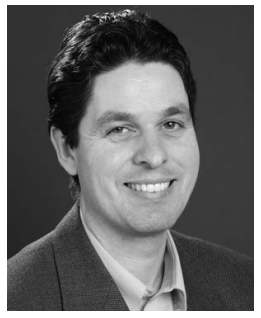

Geoffrey I. Webb holds a research chair in the Faculty of Information Technology, Monash University. Prior to this, he held appointments at Griffith University and then Deakin University, where he was a personal chair. His primary research areas are machine learning, data mining, and user modeling. He has developed numerous algorithms and techniques for machine learning, data mining, and user modeling. His commercial data mining software, Magnum Opus, is marketed internationally by Rulequest Research. He is the editor in chief of the highest impact data mining journal, Data Mining and Knowledge Discovery, and a member of the editorial boards of Machine Learning, the ACM Transactions on Knowledge Discovery in Data, and User Modeling and User-Adapted Interaction. $\mathrm{He}$ is a senior member of the IEEE.

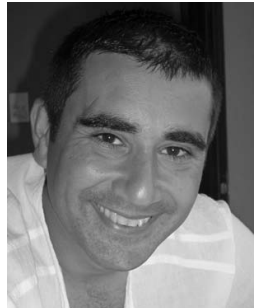

Jesús Cerquides received the $\mathrm{PhD}$ degree in artificial intelligence from the Univesitat Politècnica de Catalunya, Spain, in 2003. After being an associate director at UBS AG, Switzerland, and the chief technology officer at Intelligent Software Components (iSOCO), Spain, he is currently an associate professor at the University of Barcelona, Spain. He is an active researcher in the fields of machine learning and electronic commerce, more specifically into Bayesian classification, graphical models, auctions, and supply chain formation.

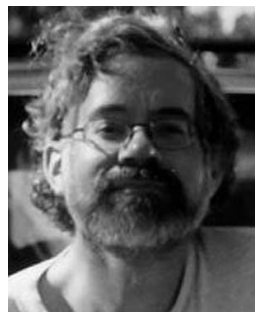

Kevin B. Korb received the PhD degree in philosophy of science from Indiana University in 1992, where he investigated the philosophical and computational foundations of scientific induction. $\mathrm{He}$ is a reader in the Clayton School of Information Technology, Monash University, Australia. His current research continues to investigate joint problems in machine learning and the philosophy of science, especially the automation of causal discovery (learning Bayesian networks). He currently leads research projects on evolutionary artificial life simulations, applications of Bayesian network modeling to meteorology, ecological systems, epidemiology, poker, and so forth, the causal interpretation of Bayesian nets, the theory of machine learning evaluation, informal logic, and argumentation. $\mathrm{He}$ is a coauthor (with Ann Nicholson) of Bayesian Artificial Intelligence, published by Chapman Hall/CRC in 2004. He was a cofounder of Psyche: An Interdisciplinary Journal of Research on Consciousness.

Janice Boughton is a research associate in the Faculty of Information Technology, Monash University. Her primary area of research is seminaive Bayesian learning.

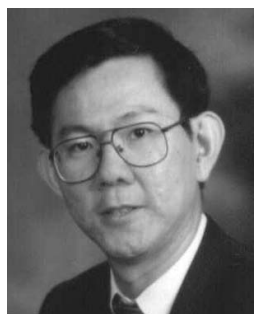

Kai Ming Ting received the PhD degree from the University of Sydney, Australia. He worked at the University of Waikato, New Zealand, and Deakin University, Australia. He has been with Monash University since 2001 . He is now an associate professor in the Gippsland School of Information Technology. His current research interests are in the areas of cost-sensitive learning, model evaluation methods, model combination, ant-based clustering, data mining, and machine learning in general. He is one of the three cochairs of the 12th Pacific-Asia Conference on Knowledge Discovery and Data Mining (PAKDD '08) and a member of the program committee for a number of international conferences.

$\triangleright$ For more information on this or any other computing topic, please visit our Digital Library at www.computer.org/publications/dlib. 